\title{
Index to Volume 117
}

\section{Compiled by Leslie Cody}

Abies amabilis, 213

balsamea, 72,532,590

balsamifera, 362

lasiocarpa, 40,44,61,353,362,566

Abietinaria abietina, 557

kincaidi, 561

pulchra, 559

thuiarioides, 561

Accipiter gentilis, 12,479

striatus, 12

Acer spp., 378

pensylvanicum, 535

rubrum, 185,436,469,532,590,622

saccharinum, 470

saccharum, 389,532,590,622

saccharum ssp. nigrum, 649

saccharum ssp. saccharum, 649

Acesta angolensis, 440

bullisi, 440

columbiana, 440

excavata, 440

Acesta excavata (Bivalvia: Pectinoidea: Limidae), in the Northwest Atlantic, First record of the European Giant File Clam, 440

Achillea millefolium, 198,260

Acipenser fulvescens, 541

oxyrynchus, 59

Acipenser fulvescens, in a Natural Reach of the Ottawa River, Movement of Lake Sturgeon, 541

Aconitum delphinifolium ssp. paradoxum, 278

Actaea pachypoda, 650 rubra, 650

Actinemys, 415

Actitis macularia, 13

Adams, J.D., 415

Adiantum pedatum, 650

Adoxa moschatellina, 278,448

Aechmophorus occidentalis, 11

Aegolius acadicus, 14 funereus, 14

Aethia cristatella, 14 psittacula, 13

Agelaius phoeniceus, 16,169 tricolor, 311

XAgrohordeum macounii, 283

Agropyron cristatum, 168 dasystachum, 262 intermedium, 283 pectiniforme, 280 sibiricum, 280 smithii, 25,168,262 subsecundum, 262

Agrostis alba, 168

borealis, 452

capillaris, 536

exarata, 278 gigantea, 280

mertensii ssp. mertensii, 278,448

palustris, 536

scabra, 535

scabra var. geminata, 278

stolonifera, 536

tenuis, 536

Aix sponsa, 608

Alaska, A Northern Hawk Owl, Surnia ulula, Nest on a Manmade Structure in, 306

Alaska, Heavy Metal Concentrations in Arctic Foxes, Alopex lagopus, in the Prudhoe Bay Oil Field, 119

Albatross, Black-footed, 11

Alberta, Documenting Pronglorn Antelope, Antilocapra americana, in the Peace River Grasslands, 657

Alberta, Northern Harrier, Circus cyaneus, Attacks on Greater Sage-Grouse, Centrocercus urophasianus, in Southern, 479

Alberta, Seasonal Dynamics and Defoliation Impact on Herbage Yield in Aspen Boreal Habitats of, 196

Alces alces, 7,316,357,400,547,591

alces andersoni, 7

alces gigas, 7

Alder, Green, 203,290,455

Grey, 290

Sitka, 215

Speckled, 378,417

Aldridge, C.L., 479

Aldridge, C.L. and R.M. Brigham. Distribution, Abundance, and Status of the Greater Sage-Grouse, Centrocercus urophasianus, in Canada, 25

Alectoria, 39,44

ochroleuca, 66

sarmentosa, 39,46,62,354

Alewife, 59

Alfalfa, Yellow, 459

Alldredge, A.W., 316

Allium tricoccum, 650

Alnus crispa, 203,215,448

crispa ssp. crispa, 278

incana, 417

incana ssp. tenuifolia, 178

rubra, 213

rugosa, 378

Alopecurus geniculatus, 278

Alopex lagopus, $119,424,430$

Alopex lagopus, Energy Cost of Running in an Arctic Fox, 430

Alopex lagopus, in the Prudhoe Bay Oil Field, Alaska, Heavy Metal Concentrations in Arctic Foxes, 119

Alosa pseudoharengus, 59 sapidissima, 59

Alvo, R. and S. Ponomarenko. Vegetation Classification Standard for Canada Workshop: 31 May-2 June 2000, 125

Amanita, 380 
Ambrosia psilostachya, 645

Ambystoma jeffersonianum, 19

Ammodramus maritimus, 654 nelsoni, 169

Ammodytes hexapterus, 53

Amphibian Voice, 166,677

Amphicarpa bracteata, 650

Amphipacifica: Journal of Aquatic Systematic Biology 3(3), 164

Anas acuta, 12,605

americana, 11

clypeata, 12

crecca, 12

cyanoptera, 12

discors, 12,227,314

penelope, 11

platyrhynchos, 11,254

rubripes, 11

strepera, 11,608

Anas acuta, Initiate Rapid Follicular Growth During Spring Migration?, Do Female Northern Pintails, 605

Anderson, E.M., 419

Anderson, S.H., 308

Andromeda polifolia, 278

Andropogon gerardii, 218 hallii, 645

Androsace septentrionalis, 262

Anemone acutiloba, 650

canadensis, 103

multifida, 278

patens, 260

Anemone, Cut-leaf, 291

Angelica lucida, 449

Angelica, Seacoast, 461

Anguilla rostrata, 59

Anser albifrons, 11

Antelope, Antilocapra americana, in the Peace River Grasslands, Alberta, Documenting Pronglorn, 657

Antelope, Pronglorn, 657

Antennaria rosea ssp. confinis, 278

Anthus rubescens, 15

Antilocapra americana, 309,657

Antilocapra americana, in the Peace River Grasslands, Alberta, Documenting Pronglorn Antelope, 657

Aoki, K.A.A. and R.A. Bodaly. Sympatric Presence of Low and High Gillraker Forms of Cisco, Coregonus artedi, in Lake Athapapuskow, Manitoba, 49

Apargidium, 214

Aphelocoma californica, 311

Aphriza virgata, 13

Apocynum cannabinum var. glaberrimum, 449 sibiricum, 462

Apodemus sylvaticus, 245

Applegate, D.R., 122

Applegate, R.D., 645

Applegate, R.D. and D.R. Applegate. Winter Occurrence of Harlan's, Buteo jamaicensis harlani, and Krider's, $B$. j. borealis, Hawks in Eastern Kansas, 122

Aptenodytes forsteri, 231,430

Aquadro, C.F., 184

Aquila chrysaetos, $12,31,479,566$

Aquilegia formosa, 278

Arabis boivinii, 278

codyi, 278

holboellii var. retrofracta, 279 kamtschatica, 279

Aralia spp., 594

Arceuthobium campylopodum, 306

Archibold, O.W., E.A. Ripley, and L. Delanoy. Effects of Season of Burning on the Microenvironment of Fescue Prairie in Central Saskatchewan, 257

Arcoscalpellum mitchellotianum, 441

Arctagrostis latifolia, 449

Arctanthemum integrifolium, 66

Ardea alba, 11 herodias, 11,603

Arenaria interpres, 13 melanocephala, 13

Arnica angustifolia ssp. attenuata, 279

chamissonis ssp. chamissonis, 279

griscomii ssp. frigida, 279

Arnica, Alpine, 299

Meadow, 299

Aronia melanocarpa, 395

Arrowhead, 168

Arsenault, A., 61

Artemisia borealis, 463

campestris, 449

cana, 25

dracunculus, 279

filifolia, 645

frigida, 25,262

ludoviciana, 258

tilesii, 449

Arthurs, W., Review by, 669

Ash, Black, 590

Green, 218

White, 649

Asio flammeus, 14,466

Aspen, 362,582

Largetooth, 436

Trembling, 76,91,196,378,395,436,590,652

Aspen Boreal Habitats of Alberta, Seasonal Dynamics and Defoliation Impact on Herbage Yield in, 196

Asphodel, Sticky False, 213

Aster falcatus, 258

laevis, 103,258

modestus, 279

sagittifolius, 198

sibiricus, 449

spathulatus, 449

umbellatus, 538

Aster, 258,463

Arctic, 463

Western Bog, 299

Astragalus aboriginum, 459

australis, 449

bodinii, 279

cicer, 280

goniatus, 262

tenellus, 279

Athyrium filix-femina var. angustum, 650

Atkinson, J., Reviews by, 158,508,674

Auditor's Report, 518

Auklet, Cassin's, 13

Crested, 14

Parakeet, 13

Rhinoceros, 14

Aulacomnium turgidum, 66

Aulichnites, 578 
Avens, Hooker's Mountain, 294

Three-flowered, 258

Awlwort, 294,458

Aythya affinis, 12,582

americana, 12

collaris, 12,585,608

marila, 12,582

valisineria, 12,608

Aythya affinis, Reproductive Rates Declined in Parkland Manitoba?, Have Lesser Scaup, 582

Azalea, False, 214

Badger, 308

American, 348

Eurasion, 243

Bailey, M., Review by, 660

Balanus improvisus, 555

Ballard, W.B., 424,468,645

Ballard, W.B., M.A. Cronin, M.D. Robards, and W.A. Stubblefield. Heavy Metal Concentrations in Arctic Foxes, Alopex lagopus, in the Prudhoe Bay Oil Field, Alaska, 119

Baneberry, Red, 650

White, 650

Bartramia longicauda, 13

Basswood, 590

American, 650

Bat, Big Brown, 652

Eastern Red, 651

Hoary, 652

Little Brown, 17,652

Silver-haired, 652

Bat, Lasiurus borealis, from Cypress Hills Provincial Park, Saskatchewan: A Response to Climate Change?, New Records of the Eastern Red, 651

Bear, American Black, 18

Black, 113,193,407,566,591,621,626

Brown, 242

Florida Black, 113

Grizzly, 18,566

Louisiana Black, 113

North American Black, 236

Plains Grizzly, 30

Polar, 433,648

Svalbard Polar, 120

Bear, Ursus americanus, Denning Chronology and Den Site Selection in the Northeastern Cascades of Washington, Black, 626

Bear, Ursus maritimus, in northeast Saskatchewan, Extralimital Sighting of a Polar, 648

Bears, Ursus americanus, Associated with Elevated Train Trestles, Mortality of Black, 113

Bears, Ursus americanus, in Difficult Locations, Methods for Capturing Free-Ranging, 621

Bears, Ursus americanus, Phenotypic Variation in Skull Size and Shape Between Newfoundland and Mainland Populations of North American Black, 236

Beaudoin, A.B., Review by, 670

Beaver, 115,192,365,378,590

American, 17

Bedstraw, Northern, 258 Small, 298

Beech, 185,394,538,590 American, 389

Beggarticks, Nodding, 299
Bélanger, L., 167

Belant, J.L. and S.K. Windels. Small Mammal Abundance and Diversity in Forests with and without Canada Yew, Taxus canadensis, 389

Bell, Yellow, 304

Bellwort, Large-flowered, 650

Bennett, B., 278

Bennett, R., Review by, 674

Bergenske, J., 352

Bernier, C., 190

Betula spp., 395

alleghaniensis, 532,590

cordifolia, 532

glandulosa, 203,307

lenta, 622

neoalaskana, 279

occidentalis, 279

papyrifera, $61,279,362,378,532,590$

pendula, 393

populifolia, 393

Betula pendula, on the Hydrology of Wainfleet Bog, Ontario, Lack of Evidence for Impact of the European White Birch, 393

Bidens cernua, 279

Bider, J.R., 377

Bimeria brevis, 561

Birch, 395

Alaska, 290

Black, 622

Bog, 307

Dwarf, 203

European White, 393

Mountain, 532

Paper, 290,362

Water, 290

White, 378,532,590

Yellow, 532,590

Birch, Betula pendula, on the Hydrology of Wainfleet Bog, Ontario, Lack of Evidence for Impact of the European White, 393

Bishop's Cap, Two-leaved, 650

Bison bison, 18,657

Bison, 657

American, 7

Bitterbrush, 628

Bitter-cress, Little Western, 292

Bittern, American, 11

Blackbird, Red-winged, 7,169

Rusty, 16

Tricolored, 311

Bladderpod, Arctic, 294

Bladderwort, Lesser, 298

Blarina brevicauda, 246,390

Blechnum spicant, 211

Blue-joint, 282

Blueberry, Bog, 213

Oval-leaved, 461

Bluebird, Mountain, 7,110

Bluebirds, Sialia currucoides, and Tree Swallows, Tachycineta bicolor with Monthly Air Temperatures, Comparison of Spring Return Dates of Mountain, 110

Bluegrass, 196

Bog, 285

Canada, 285

Kentucky, 168,196,258 
Rough, 285

Sandberg, 285

Bluestem, Big, 218

Little, 218

Old World, 425

Sand, 645

Bobcat, 193,361,472

Bobcats, Lynx rufus, and Raccoons, Procyon lotor, Relative to Home Range Boundaries, Capture Locations of Coyotes, Canis latrans, 472

Bobolinks, 169

Bodaly, R.A., 49

Boertmann, D. Distribution and Conservation of the Harlequin Duck, Histrionicus histrionicus, in Greenland, 249

Bog-laurel, 296

Boles, S.R., 469

Boletus, 380

Bombycilla cedrorum, 15,177 garrulus, 15

Bonasa umbellus, 12,177,313,365

Boreal Dip Net: Newsletter of the Canadian Amphibian and Reptile Conservation Network, The, 343

Bork, E.W., 196

Botaurus lentiginosus, 11

Bothriochloa spp., 425

Botrychium lunaria, 449

Bougainvillia sp., 557

Bourgeois, J.-C., 377,605

Bousfield, E.L., 140

Bouteloua curtipendula, 218,425 gracilis, 25,425,645

Brachyramphus brevirostris, 13 marmoratus, 13,53

Bramble, Five-leafed, 214

Brambling, 16

Brant, 11

Branta bernicla, 11 canadensis, 11,227

Breton, L., 399,546

Brigham, R.M., 25,651

British Columbia, and its Palaeobotanical Implications, An Occurrence of the Hawkweed-Leaved Saxifrage, Saxifraga hieraciifolia, in Southern, 304

British Columbia, Bald Eagles, Haliaeetus leucocephalus, Feeding on Spawning Plainfin Midshipman, Porichthys notatus, at Crescent Beach, 601

British Columbia, Characteristics of Early-Winter Caribou, Rangifer tarandus caribou, Feeding Sites in the Southern Purcell Mountains, 352

British Columbia Coast, The Shoreline Fringe Forest and Adjacent Peatlands of the Southern Central, 209

British Columbia, Notes on the Populus "Dripzone Effect" on Lichens in Well-ventilated Stands in East-central, 61

British Columbia, Seasonal Habitat Use and Movements of Mountain Goats, Oreamnos americanus, in East-central, 565

British Columbia, Sightings of Vagrant Pacific Alcids in Desolation Sound, 53

Brome, California, 282 Smooth, 168,197

Bromegrass, 196 Smooth, 196

Bromus carinatus, 280 inermis, 168,196

secalinus, 536

Brooks, R.T., 190

Browning, M., 393

Brunjes, J.H. IV and W.D. Webster. Marsh Rice Rat, Oryzomys palustris, Predation on Forster's Tern, Sterna forsteri, Eggs in Coastal North Carolina, 654

Brunton, D.F. A Tribute to Clarence Frankton 1906-2000, 481

Bryoria spp., 39,44,352

capillaris, 45,62

chalybeiformis, 45

fremontii, 39,45

fuscescens, 39,45,62

glabra, 39,45

implexa, 45

lanestris, 45

pseudofuscescens, 39,45

simplicior, 45

tortuosa, 45

(Bryoria) in High-Elevation Oldgrowth Conifer Forests, On the Dispersal of Hair Lichens, 44

(Bryoria) in the Canopies of High-elevation Oldgrowth Conifer Forests, On the Vertical Zonation of Hair Lichens, 39

Bubo virginianus, 14,227,479

Bucephala albeola, 12 clangula, 12 islandica, 12

Buchloe dactyioides, 425,645

Buckbean, 297,436,462

Buckleberry, 352

Budworm, Spruce, 531

Buffalograss, 425

Bufflehead, 12

Bufo americanus, 380,477

Bugseed, 455

Bulrush, 103

Common Great, 453

Hardstem, 226

Bunchberry, 214,352

Bunting, Snow, 7,177

Bur-reed, Giant, 168 Small, 281

Burbot, 59

Burke, A.M. Sandhill Crane, Grus canadensis, Nesting in the Yorkton Wetland Complex, Saskatchewan, 224

Burnet, Canadian, 295 Great, 213

Bush, Flowering, 103

Buteo jamaicensis, 12,122,179,479

jamaicensis borealis, 122

jamaicensis harlani, 122

lagopus, 12

regalis, 479

swainsoni, 12,479

Buteo jamaicensis borealis, Hawks in Eastern Kansas, Winter Occurrence of Harlan's, Buteo jamaicensis harlani, and Krider's, 112

Buteo jamaicensis harlani, and Krider's, B. j. borealis, Hawks in Eastern Kansas, Winter Occurrence of Harlan's, 122

Butomus umbellatus, 103

Butter-and-eggs, 298

Buttercup, Birdfoot, 292 
Dwarf, 457

Pallas', 457

Snow, 457

Subalpine, 292

Sulpher, 457

White Water, 292

Butterweed, Rayless Mountain, 299

Cabanac, A.J. Physical Condition of an Animal, Using as an Example the Common Eider, Somateria mollissima, 230

Cabbage, Deer, 213

Cadorette, S., Review by, 662

Calamagrostis canadensis, 168,535

canadensis ssp. canadensis, 279

canadensis ssp. langsdorfii, 279

purpurascens var. purpurascens, 279 stricta ssp. inexpansa, 279

Calamovilfa longifolia, 645

Calcarius lapponicus, 16 pictus, 16

Calder, D.R. Subtidal Hydroids (Cnidaria) of Northumberland Strait, Atlantic Canada, with Observations on Their Life Cycles and Distributions, 555

Calidris alba, 13

alpina, 13

bairdii, 13

canutus, 13

mauri, 13

melanotos, 13

minutilla, 13

ptilocnemis, 13

pusilla, 13

ruficollis, 13

Caligus clemensi, 634

Caloplaca holocarpa, 62

Caltha natans, 449 palustris ssp. arctica, 449

Calycella syringa, 557

Calypte anna, 14

Calyptospadix cerulea, 561

Campanula rotundifolia, 260

Campanularia gelatinosa, 561 groenlandica, 557

hincksii, 561

volubilis, 559

Campbell, C.E., 477

Campbell, G.D., 589

Campion, Arctic, 291

Bladder, 260

Moss, 291

Taimyr, 291

Canada*, A Review of the Canada Lynx, Lynx canadensis, in, 360

Canada, Distribution, Abundance, and Status of the Greater Sage-Grouse, Centrocercus urophasianus, in, 25

Canada, Dryopteris goldiana $\times D$. intermedia, a Natural Fern Hybrid New to, 649

Canada Lynx, Lynx canadensis, in Canada*, A Review of the, 360

Canada, Malaxis monophyllos var. brachypoda, One-leaved Malaxis, new to the Northwest Territories, 302

Canada, Patterns of Nestling Feeding in Harris's Sparrows, Zonotrichia querula and White-crowned Sparrows, Z. leucophrys, in the Northwest Territories, 203
Canada, Supposed Periodicity of Redpoll, Carduelis sp., Winter Visitations in Atlantic, 611

Canada, II, Additions and Range Extensions to the Vascular Plant Flora of the Continental Northwest Territories and Nunavut, 448

Canada, with Observations on Their Life Cycles and Distributions, Subtidal Hydroids (Cnidaria) of Northumberland Strait, Atlantic, 555

Canada Workshop: 31 May-2 June 2000, Vegetation Classification Standard for, 125

Canada Yew, Taxus canadensis, Small Mammal Abundance and Diversity in Forests with and without, 389

Canadian Atlantic Ichthyofauna, The Chain Dogfish, Scyliorhinus retifer (Garman, 1881), New to the, 475

Canadian Field-Naturalist, Advice for Contributors to The, 522

Canadian Species at Risk, 166,513

Canis familiaris, 123,380

Canis latrans, 17,30,123,242,308,364,468,472,645

latrans-lupus, 589

lupus, $17,30,120,242,357,364,400,419,468,469,550,566$

lupus ligoni, 7

lupus nubilus, 7

lupus occidentalis, 7

lupus X latrans, 589

lupus-lycaon, 589

lycaon, 589

mesomelas, 424

rufus, 589

Canis familiaris, Kill Coyote, Canis latrans, Feral Dogs, 123

Canis latrans, Bobcats, Lynx rufus, and Raccoons, Procyon lotor, Relative to Home Range Boundaries, Capture Locations of Coyotes, 472

Canis latrans, Feral Dogs, Canis familiaris, Kill Coyote, 123

Canis latrans - Rio Grand Turkey, Meleagris gallopavo intermedia, interactions, Coyote, 645

Canis lupus, in Porcupine, Erethizon dorsatum, Dens in Wisconsin, Death of Gray Wolves, 469

Canis lupus, Pack Structure: Conservation Implications for Recovering Populations, Limits to Plasticity in Gray Wolf, 419

Canis lupus $\times$ latrans Hybrid Zone, Southeastern Ontario, Landscape Influence on Canis Morphological and Ecological Variation in a Coyote-Wolf, 589

Canvasback, 12,608

Capelin, 70

Capreolus capreolus, 242

Cardamine oligosperma ssp. kamtschatica, 292 umbellata, 279

Cardinal, Northern, 177

Cardinalis cardinalis, 177

Carduelis sp., 611

flammea, 16,611

hornemanni, 16,611

pinus, 16,177

tristis, 179

Carduelis sp., Winter Visitations in Atlantic Canada, Supposed Periodicity of Redpoll, 611

Carex spp., 103,210

arcta, 279,449

atherodes, 103,279

atrofusca, 279

bicolor, 279

bonanzensis, 279

brunnescens, 279 
buxbaumii, 279

capillaris ssp. capillaris, 279

concinna, 279

deflexa, 279

disperma, 449

eleocharis, 262

eleusinoides, 279

filifolia, 197

flava, 279

franklinii, 279

garberi ssp. bifaria, 279

glacialis, 279

heliophila, 258

hoodii, 448

lanuginosa, 103

leporina, 536

magellanica ssp. irrigua, 279

maritima, 449

media, 279

microglochin, 279

microptera, 448

misandra, 67

nardina, 67

obtusata, 258,279

pachystachya, 279

pauciflora, 214

pedunculata, 650

petasata, 448

phaeocephala, 279

plantaginea, 650

platyphylla, 650

rostrata, 103,279

scirpoidea, 67

tenuiflora, 449

trisperma, 537

viridula, 279

williamsii, 279

Caribou, 18,120,253,399

Barren-ground, 7,39

Mountain, 39,44,356

Woodland, 7,39,44,352,546

Caribou, Rangifer tarandus caribou, An Aerial Survey Technique for the Forest-Dwelling Ecotype of Woodland, 546

Caribou, Rangifer tarandus caribou, Feeding Sites in the Southern Purcell Mountains, British Columbia, Characteristics of Early-Winter, 352

Caribou, Rangifer tarandus, in Quebec, Historical Changes and Current Distribution of, 399

Carlson, J., W.A. Gough, J.D. Karagatzides, and L.J.S. Tsuji. Canopy Interception of Acid Deposition in Southern Ontario, 523

Carpodacus purpureus, 16

Carya spp., 19,622 cordiformis, 218,649

Cassiope mertensiana, 354 tetragona, 66

Castilleja caudata, 298 pallida var. caudata, 279

Castor canadensis, 17,115,192,365,378,590

Cat, Domestic, 380 Feral, 380

Catastomous catostomous, 59

Cathartes aura, 11

Catharus guttatus, 15 minimus, 15

ustulatus, 15

Catling, P.M. Dryopteris goldiana $\times$ D. intermedia, a Natural Fern Hybrid New to Canada, 649

Catostomous commersoni, 59

Cattail, 103,168

Caulophyllum thalictroides, 650

Cedar, Eastern White, 590

Western Red, 214

White, 470

Yellow, 213

Celtis occidentalis, 218

Centrocercus spp., 25 urophasianus, 25,308,479

Centrocercus urophasianus, in Canada, Distribution, Abundance, and Status of the Greater Sage-Grouse, 25

Centrocercus urophasianus, in Southern Alberta, Northern Harrier, Circus cyaneus, Attacks on Greater SageGrouse, 479

Centrocercus urophasianus, Nest Predators Using Remote Sensing Cameras, Direct Identification of Northern Sage-grouse, 308

Cephaloziella spp., 535

Cepphus columba, 13,54

Cerastium alpinum, 67 arvense, 260,279

Ceratophyllum demersum, 279

Cerorhinca monocerata, 14

Certhia americana, 15,177

Cervus elaphus, 196,309,357 elaphus nelsoni, 316

Cervus elaphus nelsoni, During the Calving Season Toward Mule Deer, Odocoileus hemionus, in Central Colorado, Aggressive Interactions of Rocky Mountain Elk, 316

Ceryle alcyon, 14

Chaetura vauxi, 14

Chamaecyparis nootkatensis, 212

Chamaedaphne calyculata, 436,449

Chamberlain, M.J., 113

Chamerion latifolium, 67

Char, Arctic, 59,254,634

Chara spp., 273

Charadrius semipalmatus, 12 vociferus, 11,311

Chardine, J.W., 70

Chelydra serpentina serpentina, 415

Chen caerulescens, 11,226 caerulescens atlantica, 608 caerulescens caerulescens, 608 canagica, 11

Cherry, Black, 19,395

Chickadee, Black-capped, 15,179

Boreal, 15

Chestnut-backed, 7

Gray-headed, 15

Mountain, 15,76

Chickadees, Poecile gambeli, to Playback of Different Song Types, Response of Male Mountain, 76

Chickweed, 291

Field, 260,290

Long-leaved, 291

Chiloscyphus spp., 535

Chinook, 639

Chipmunk, Eastern, 246,591

Least, 7,309 
Chlidonias niger, 13

Choke-cherry, 417

Chondestes grammacus, 16

Chordeiles minor, 14

Chorilia longipes, 118

Choristoneura fumiferana, 531

Chrysanthemum integrifolium, 66,279,449 leucanthemum, 536

Chrysemys picta picta, 415

Chrysosplenium tetrandrum, 449

Chrysothamnus sp., 645

Chub, Lake, 59

Chum, 638

Cicuta maculata var. angustifolia, 279

Cinclus mexicanus, 15

Cinna latifolia, 279

Cinquefoil, Snow, 295

Three-toothed, 459

White, 260

Circaea alpina ssp. alpina, 279

Circus cyaneus, 12,227,479

Circus cyaneus, Attacks on Greater Sage-Grouse, Centrocercus urophasianus, in Southern Alberta, Northern Harrier, 479

Cirriped, Stalked, 441

Cirsium spp., 103

arvense, 198,536

vulgare, 536

Cisco, 49

$$
\text { Shortjaw, 49,642 }
$$

Cisco, Coregonus artedi, in Lake Athapapuskow, Manitoba, Sympatric Presence of Low and High Gillraker Forms of, 49

Cistothorus palustris, 169,348,654

Cladina mitis, 66

Cladonia spp., 352,535

Cladonia, 352

Clam, Acesta excavata (Bivalvia: Pectinoidea: Limidae), in the Northwest Atlantic, First record of the European Giant File, 440

Clam, European Giant File, 440

Clangula hyemalis, 12,254

Clava leptostyla, 561 multicornis, 561

Clemmys, 415 guttata, 436 insculpta, 377

Clemmys guttata, Near their Northern Limit: an 18-Year Follow-up, Population Structure, Growth, and Age Estimation of Spotted Turtles, 436

Clethrionomys spp., 246,348 gapperi, 246,390 rutilus, 17

Clover, 168

Red, 296

White, 197

White Sweet, 459

Club-moss, Alpine, 280

Common, 280,450

Flatbranch, 280,450

Running, 214

Clubrush, Tufted, 213,288

Clytia hemisphaerica, 559

Coad, B.W., 475

Coad, B.W., Reviews by, 151,318,319,323,324
Coccothraustes vespertinus, 177

Cody, W.J., Reviews by, 329,330,496,669

Cody, W.J. and V. Johnston. Malaxis monophyllos var. brachypoda, One-leaved Malaxis, new to the Northwest Territories, Canada, 302

Cody, W.J., C.E. Kennedy, B. Bennett, and J. Staniforth. New Records of Vascular Plants in the Yukon Territory V, 278

Cody, W.J., K.L. Reading, and J.M. Line. Additions and Range Extensions to the Vascular Plant Flora of the Continental Northwest Territories and Nunavut, Canada, II, 448

Coelopleurum gmelinii, 461

Coho, 638

Cohosh, Blue, 650

Colaptes auratus, 14

Colorado, Aggressive Interactions of Rocky Mountain Elk, Cervus elaphus nelsoni, During the Calving Season Toward Mule Deer, Odocoileus hemionus, in Central, 316

Coltsfoot, Arrow-leaved, 464

Sweet, 464

Columba fasciata, 14 livia, 14,176

Columbine, Western, 291

Comandra umbellata ssp. pallida, 279

Comandra, Pale, 290

Contopus cooperi, 14 sordidulus, 14

Cook, F.R., Reviews by, 490,491,492,493,494,495,509

Cooke, S.J., Reviews by, 156,499

Coot, American, 12

Coral, 440

Corallorhiza trifida, 449

Coralroot, Yellow, 454

Cordgrass, Smooth, 654

Cordylophora caspia, 561 lacustris, 561

Coregonus, 49 artedi, 49

clupeaformis, 59,642 zenithicus, 49,642

Coregonus artedi, in Lake Athapapuskow, Manitoba, Sympatric Presence of Low and High Gillraker Forms of Cisco, 49

Corispermum hookeri, 448 ochotense, 448

orientale var. emarginatum, 455 villosum, 448

Cormorant, Double-crested, 11 Pelagic, 11

Cornus canadensis, 211,352,535

Corvus brachyrhynchos, 30,227 caurinus, 15,602 corax $, 15,30,227,308$

Corymorpha pendula, 558

Cosgrove, J.A. An in situ Observation of Webover Hunting by the Giant Pacific Octopus, Enteroctopus dofleini (Wülker, 1910), 117

Cotton-grass, 395

Arctic, 288

Scheuchzer's, 288

Short-anthered, 287

Cottontail, 314

Eastern, 591,645 
Cottus bairdi, 59 cognatus, 59

Coturnix japonica, 348

Couesius plumbeus, 59

Cougar, 7,357,566

Courtois, R., A. Gingras, C. Dussault, L. Breton and J.-P. Ouellet. An Aerial Survey Technique for the ForestDwelling Ecotype of Woodland Caribou, Rangifer tarandus caribou, 546

Courtois, R., J.-P. Ouellet, A. Gingras, C. Dussault, L. Breton and J. Maltais. Historical Changes and Current Distribution of Caribou, Rangifer tarandus, in Quebec, 399

Couture, R., 605

Cow, Domestic, 309

Cowbird, Brown-headed, 16,348

Coyote, 17,30,123,227,242,308,364,468,472,645

Coyote, Canis latrans, Feral Dogs, Canis familiaris, Kill, 123

Coyote, Canis latrans - Rio Grand Turkey, Meleagris gallopavo intermedia, interactions, 645

Coyote-Wolf, 589

Coyote-Wolf, C. lupus $\times$ latrans Hybrid Zone, Southeastern Ontario, Landscape Influence on Canis Morphological and Ecological Variation in a, 589

Coyotes, Canis latrans, Bobcats, Lynx rufus, and Raccoons, Procyon lotor, Relative to Home Range Boundaries, Capture Locations of, 472

Crab, 117

Crab-Apple, Pacific, 215

Craig, T., 306

Cranberry, Bog, 213,461

Large, 436

Small, 436

Crane, Sandhill, 12,224

Whooping, 224

Crane, Grus canadensis, Nesting in the Yorkton Wetland Complex, Saskatchewan, Sandhill, 224

Crassostrea virginica, 555

Crassula aquatica, 449

Crataegus sp., 311

Creeper, Brown, 7,177

Crepis tectorum, 448

Cress, Garden, 294

Crocus, Prairie, 260

Cronin, M.A., 119

Crossbill, Red, 16 White-winged, 16

Crow, American, 30,227 Northwestern, 7,602

Crowberry, 213

Curatorial Reports on Natural History Topics: Nova Scotia Museum of Natural History, 165

Curlew, Bristle-thighed, 13

Cuspidella humilis, 557

Cyanocitta cristata, 179,193 stelleri, 15

Cyclopterus lumpus, 254

Cygnus buccinator, 11 columbianus, 11

Cyperus esculentus, 106 rotundus, 106

Cystophora cristata, 121

Cystopteris fragilis, 279,449 montana, 279,449
Dace, Longnose, 57

Pearl, 59

Dace, Rhinichthys cataractae, in Newfoundland and Labrador, Range Extensions of Logperch, Percina caprodes, and Longnose, 57

Dactylis glomerata, 278

Daigle, C., 377

Daisy, Entire-leaved, 66,299,463

Daisy, Hulteniella integrifolia (Asteraceae), in Québec, Ecogeographical and Cytological Notes on the Entireleaved, 66

Danby, R.K. Birds and Mammals of the St. Elias Mountain Parks: Checklist Evidence for a Biogeographic Convergence Zone, 1

Dandelion, 197

Danthonia intermedia, 449 spicata, 448

Davies, J.-A., 352

Deer, European Roe, 242

Mule, 7,309,316,357,657

Red, 309

White-tailed, 309,316,357,389,407,567,590

Deer, Odocoileus hemionus, in Central Colorado, Aggressive Interactions of Rocky Mountain Elk, Cervus elaphus nelsoni, During the Calving Season Toward Mule, 316

DeGraaf, R.M., 190

Delanoy, L., 257

Delphinium glaucum, 279

Dendragapus obscurus, 12

Dendranthema integrifolium, 66

Dendroica caerulescens, 389

coronata, 15

palmarum, 15

petechia, 15,206,309

striata, 15

townsendi, 15

Dennstaedtia punctilobula, 535

Deparia acrostichoides, 650

Deschampsia elongata, 448

Descurainia incisa var. incisa, 448 sophioides, 279

Desmognathus fuscus, 19 ochrophaeus, 19

Diamond, J., M. Browning, A. Williams, and J. Middleton. Lack of Evidence for Impact of the European White Birch, Betula pendula, on the Hydrology of Wainfleet Bog, Ontario, 393

Dicoryne conferta, 558

Dicranum sp., 214

elongatum, 66

ontariense, 535

Dicrostonyx, 466

Dignard, N. and C. Gervais. Eco-geographical and Cytological Notes on the Entire-leaved Daisy, Hulteniella integrifolia (Asteraceae), in Québec, 66

Dion, N., K.A. Hobson and S. Larivière. Does Removal of Duck Nest Predators Affect the Temporal Patterns of Predation for Simulated Nests of Grassland Songbirds?, 347

Diphasia fallax, 557 kincaidi, 561

Diplazium pycnocarpon, 650

Dipper, American, 15

Dobony, C.A., 313 
Dock, Western, 103

Dockrill, C.W., 479

Dodecatheon pulchellum, 304

Dog, 380 Feral, 123

Dogbane, Common, 462

Dogfish, Chain, 475

Dogfish, Scyliorhinus retifer (Garman, 1881), New to the Canadian Atlantic Ichthyofauna, The Chain, 475

Dogs, Canis familiaris, Kill Coyote, Canis latrans, Feral, 123

Dolichonyx oryzivorus, 169

Dombrowski, P., J.-C. Bourgeois, R. Couture, and C. Linard. Do Female Northern Pintails, Anas acuta, Initiate Rapid Follicular Growth During Spring Migration?, 605

Donkor, N.T., M.M. Okello, R.J. Hudson and E.W. Bork. Seasonal Dynamics and Defoliation Impact on Herbage Yield in Aspen Boreal Habitats of Alberta, 196

Doolittle, C.J.T., 469

Dove, Mourning, 14,177 Rock, 14,176

Dowitcher, Long-billed, 13 Short-billed, 13

Draba albertina, 279 alpina, 449 borealis, 279 cana, 279 cinerea, 279,449

glabella, 449

incerta, 279

kananaskis, 278,293

lactea, 449

macounii, 279

porsildii, 279

scotteri, 279

stenoloba, 448

Draba, Alpine, 457

Macoun's, 294

Northern, 293

Slender, 293

Dropseed, 645

Sand, 425

Drosera rotundifolia, 213

Drury, C.F., 267

Dryas crenulata, 279

hookeriana, 279

integrifolia, 66

Dryopteris campyloptera, 535

carthusiana, 651

celsa, 650

clintoniana, 650

clintoniana $\times$ goldiana, 651

correllii, 651

cristata, 650

disjuncta, 451

expansa, 279

goldiana, 649

goldiana $\times$ intermedia, 651

intermedia, 649

marginalis, 390

$\times$ mickelii, 651

spinulosa, 535

Dryopteris goldiana $\times$ D. intermedia, a Natural Fern Hybrid New to Canada, 649
Dryopteris intermedia, a Natural Fern Hybrid New to Canada, Dryopteris goldiana $\times, 649$

Duck, American Black, 11

Harlequin, 12,249

Long-tailed, 7,254

Ring-necked, 7,585,608

Ruddy, 12

Wood, 608

Duck, Histrionicus histrionicus, in Greenland, Distribution and Conservation of the Harlequin, 249

Dunlin, 13

Dussault, C., 399,546

Dynamena pumila, 561

Eagle, Bald, 12,601

Golden, 12,31,479,566

Eagles, Haliaeetus leucocephalus, Feeding on Spawning Plainfin Midshipman, Porichthys notatus, at Crescent Beach, British Columbia, Bald, 601

Ectopleura crocea, 561

larynx, 558

Editor's Report for Volume 116 (2002), 345

Edwards, J.W., 313

Edwards, M.A. and G.J. Forbes. Food Habits of Ermine, Mustela erminea, in a Forested Landscape, 245

Eedy, W., Reviews by, 160,342

Eel, American, 59

Eelgrass, 655

Egret, Great, 11

Eider, Common, 12,230,608

King, 12

Eider, Somateria mollissima, Physical Condition of an Animal, Using as an Example the Common, 230

Elderberry, Red, 538

Eleocharis acicularis, 449 pauciflora var. fernaldii 453 quinqueflora, 449

Elk, 357

American, 309

Rocky Mountain, 316

Elk, Cervus elaphus nelsoni, During the Calving Season Toward Mule Deer, Odocoileus hemionus, in Central Colorado, Aggressive Interactions of Rocky Mountain, 316

Elliott, J.E., 601

Elliott, K.H., C.L. Struik and J.E. Elliott. Bald Eagles, Haliaeetus leucocephalus, Feeding on Spawning Plainfin Midshipman, Porichthys notatus, at Crescent Beach, British Columbia, 601

Elm, American, 218

White, 590

XElyhordeum macounii, 279

Elymus canadensis, 449

elongatus ssp. ponticus, 280

glaucus, 279

hispidus, 280

junceus, 278

macounii, 283

macrourus, 279

trachycaulus ssp. andinus, 279

trachycaulus ssp. glaucus, 279

trachycaulus ssp. novae-angliae, 279

trachycaulus ssp. subsecundus, 279

trachycaulus ssp. trachycaulus, 279

trachycaulus ssp. violaceus, 279 
Elytrigia intermedia, 283

Empetrum nigrum, 211

Empidonax alnorum, 14

difficilis, 14

flaviventris, 14

hammondii, 14

minimus, 14

oberholseri, 14

Emydoidea blandingii, 415

Enhydra lutris, 3

Enteroctopus dofleini, 117

Enteroctopus dofleini (Wülker, 1910), An in situ Observation of Webover Hunting by the Giant Pacific Octopus, 117

Epilobium anagalidifolium, 449

angustifolium, 535

arcticum, 449

davuricum, 449

davuricum var. arcticum, 460

hornemannii, 279,449

leptophyllum, 449

palustre, 279,449

Eptesicus fuscus, 652

Equisetum arvense, 449

fluviatile, 103

palustre, 449

scirpoides, 449

variegatum, 449

variegatum $\mathrm{ssp}$. variegatum, 279

Eragrostis trichodes, 645

Eremophila alpestris, 15

Erethizon dorsatum, 17,469

Erethizon dorsatum, Dens in Wisconsin, Death of Gray Wolves, Canis lupus, in Porcupine, 469

Erignathus barbatus, 121

Eriophorum angustifolium, 211

brachyantherum, 279

callitrix, 279

scheuchzeri, 279

vaginatum, 395

viridi-carinatum, 449

Eritrichium splendens, 279

Ermine, 18,193,245

Ermine, Mustela erminea, in a Forested Landscape, Food Habits of, 245

Erskine, A.J. and R. McManus, Jr. Supposed Periodicity of Redpoll, Carduelis sp., Winter Visitations in Atlantic Canada, 611

Erysimum cheiranthoides, 279 coarctatum, 448

Erythronium grandiflorum, 304

Esox lucius, 59

Eudendrium album, 558

$$
\text { dispar, } 557
$$

ramosum, 557

Eupatorium rugosum, 650

Euphagus carolinus, 16

Eutamias minimus, 309

Fagus, 394 grandifolia, 185,389,535,590

Falcipennis canadensis, 12

Falco columbarius, 12 peregrinus, 12 rusticolus, 12,479 sparverius, 12

tinnunculus, 424

Falcon, Peregrine, 12

Falsebox, 352

Fauria crista-galli, 211

Felis concolor, 18,357

domestica, 380

Felwort, Marsh, 462

Ferguson, T.A. Documenting Pronglorn Antelope, Antilocapra americana, in the Peace River Grasslands, Alberta, 657

Fern, Bracken, 214

Deer, 213

Evergreen Wood, 649

Fragile, 280,450

Goldie's, 649

Mountain Bladder, 281,450

Northern Lady, 650

Northern Maidenhair, 650

Northern Wood, 281

Oak, 281,451,650

Silvery Glade, 650

Fescue, Hard, 284

Mountain Rough, 89

Plains Rough, 89,258

Red, 168,284

Richardson's, 284

Tundra, 284

Fescue Prairie in Central Saskatchewan, Effects of Season of Burning on the Microenvironment of, 257

Festuca altaica, 262

altaica ssp. hallii, 258

campestris, 89

hallii, 89

lenensis, 279

richardsonii, 279

rubra, 168,279

tenuifolia, 536

trachyphylla, 280

Filellum serpens, 559

Filion, B., 167

Finch, Purple, 7

Fir, Balsam, 72,362,532,590

Douglas, 45,91,353,362,628

Subalpine, 40,44,353,362,566

Fireweed, 538

Fisher, 193,590

Flavocetraria nivalis, 66

Fletcher, Q.E., C.W. Dockrill, D.J. Saher, and C.L. Aldridge. Northern Harrier, Circus cyaneus, Attacks on Greater Sage-Grouse, Centrocercus urophasianus, in Southern Alberta, 479

Flicker, Northern, 14

Flycatcher, Alder, 14

Dusky, 7

Hammond's, 14

Least, 14

Olive-sided, 14

Pacific-slope, 7

Scissor-tailed, 14

Yellow-bellied, 14

Foamflower, 352

Fogfruit, 650

Forbes, G.J., 245

Ford, W.M., 313 
Forget-me-not, Showy Alpine, 297

Fox, Arctic, 119,424,430

Gray, 468

Kit, $123,424,468$

Red, 17,30,123,227,308,348,365,380,424,468,472,586, 591

Swift, 30,123,424,468

Fox, Alopex lagopus, Energy Cost of Running in an Arctic, 430

Fox, Vulpes velox, White Color Phase of the Swift, 468

Foxes, Alopex lagopus, in the Prudhoe Bay Oil Field, Alaska, Heavy Metal Concentrations in Arctic, 119

Foxes, Vulpes velox, in Northwestern Texas, Den Site Activity Patterns of Adult Male and Female Swift, 424

Fragaria sp., 380 virginiana ssp. glauca, 279

Frankton 1906-2000, A Tribute to Clarence, 481

Fratercula arctica, 70 cirrhata, 14 corniculata, 14

Fratercula arctica, in North America, Status of the Largest Breeding Concentration of Atlantic Puffins, 70

Fraxinus americana, 649 nigra, 590 pennsylvanica, 218

Freedman, B., 531

Fringilla montifringilla, 16

Fritillaria pudica, 304

Frog, Chorus, 477

Green, 477

Mink, 477

Northern Leopard, 477

Wood, 477

Frog, Rana septentrionalis, from Insular Newfoundland, First Record of Mink, 477

Froglog: Newsletter of the Declining Amphibian Populations Task Force, 164,343,513,677

Fuglei, E. and N.A. Oritsland. Energy Cost of Running in an Arctic Fox, Alopex lagopus, 430

Fulica americana, 12

Fuller, T.K., 190

Fulmar, Northern, 11

Fulmarus glacialis, 11

Fumagalli, L., 82

Gadwall, 7,608

Gagnon, J.-M. and R.L. Haedrich. First record of the European Giant File Clam, Acesta excavata (Bivalvia: Pectinoidea: Limidae), in the Northwest Atlantic, 440

Gaines, W.L. Black Bear, Ursus americanus, Denning Chronology and Den Site Selection in the Northeastern Cascades of Washington, 626

Gale, Sweet, 213

Galeopsis tetrahit, 103,536

Galium boreale, 258 trifidum, 279

Galliformes, ssp., 365

Gallinago gallinago, 13,169

Gardali, T. and J.D. White. Autumn and Winter Breeding Records for the American Robin, Turdus migratorius, 311

Garveia brevis, 561 franciscana, 555

Gasterosteus aculeatus, 59

Gaultheria shallon, 211
Gavia adamsii, 11

immer, 11,254

pacifica, 11

stellata, 11

Gawn, M., Review by, 322

Gehring, J.L., 419

Gehring, T.M., B.E. Kohn, J.L. Gehring, and E.M. Anderson. Limits to Plasticity in Gray Wolf, Canis lupus, Pack Structure: Conservation Implications for Recovering Populations, 419

Gentian, Spurred, 462

Gentiana propinqua, 462

Gentianella propinqua ssp. propinqua, 449

Geothlypis trichas, 16

Gervais, C., 66

Geum triflorum, 258

Gilhen, J., 415

Gilhen, J., B.W. Coad and A. Hebda. The Chain Dogfish, Scyliorhinus retifer (Garman, 1881), New to the Canadian Atlantic Ichthyofauna, 475

Gingras, A., 399,546

Ginns, J., Review by, 328

Gipson, P.S., 123,218,645

Gipson, P.S. and J.F. Kamler. Capture Locations of Coyotes, Canis latrans, Bobcats, Lynx rufus, and Raccoons, Procyon lotor, Relative to Home Range Boundaries, 472

Giroux, J.-F., 167

Glaucomys spp., 193,365 sabrinus, 17

Gleditsia triacanthos, 218

Glyptemys, 415 insculpta, 377,415

Glyptemys insculpta, at River Denys: A Second Population for Cape Breton Island, Nova Scotia, The Wood Turtle, 415

Glyptemys insculpta, Population at the Northern Limit of its Range in Québec, Ecological Aspects of a Wood Turtle, 377

Goat, Mountain, 7,565

Goats, Oreamnos americanus, in East-central British Columbia, Seasonal Habitat Use and Movements of Mountain, 565

Godfrey, 1910-2002, A Tribute to William Earl, 140

Godwit, Bar-tailed, 13

Hudsonian, 13

Marbled, 13

Golden-Plover, American, 12 Pacific, 12

Goldeneye, Barrow's, 12 Common, 12

Goldenrod, 538

Canada, 300

Northern, 300

Stiff, 260

Goldfinch, American, 179

Gonothyraea loveni, 557

Goodyear, M.A. Extralimital Sighting of a Polar Bear, Ursus maritimus, in northeast Saskatchewan, 648

Goose, Canada, 11,227

Emperor, 11

Great White-fronted, 11

Greater Snow, 608

Lesser Snow, 608

Snow, 11,226 
Gordia, 578

Goshawk, Northern, 12,479

Gosselin, M., E.L. Bousfield, and S.D. MacDonald. A Tribute to William Earl Godfrey, 1910-2002, 140

Gough, W.A., 523

Goward, T. On the Dispersal of Hair Lichens (Bryoria) in High-Elevation Oldgrowth Conifer Forests, 44

Goward, T. On the Vertical Zonation of Hair Lichens (Bryoria) in the Canopies of High-elevation Oldgrowth Conifer Forests, 39

Goward, T. and A. Arsenault. Notes on the Populus "Dripzone Effect" on Lichens in Well-ventilated Stands in East-central British Columbia, 61

Grama, Blue, 25,425,645

Sideoats, 218,425

Grammaria abietina, 561 gracilis, 557

Grass, 213

Alpine Holy, 284

Arctic Blue, 452

Buffalo, 645

Creeping Bent, 281

Giant Reed, 103

Hair, 453

Indian, 218

June, 25

Narrow-Leafed Cotton, 213

Nuttall's Alkali, 285

Orchard, 282

Polar Alkali, 285

Red Bent, 281,452

Reed Canary, 103,168,284,452

Spreading Alkali, 285

Western Porcupine, 260

Western Rye, 283

Grass-of-Parnassus, Fringed, 294

Grebe, Horned, 11

Pied-billed, 11

Red-necked, 11

Western, 11

Greenland, Distribution and Conservation of the Harlequin Duck, Histrionicus histrionicus, in, 249

Greenling, Painted, 118

Grèf, A., J. Gilhen and J.D. Adams. The Wood Turtle, Glyptemys insculpta, at River Denys: A Second Population for Cape Breton Island, Nova Scotia, 415

Grosbeak, Evening, 177

Pine, 16

Ground-cone, 298

Groundhog, 591

Groundsel, Common, 300

Rocky Mountain, 300

Grouse, 365

Blue, 7

Red, 480

Ruffed, 12,177,313,365

Sharp-tailed, 12,227,479

Spruce, 12

Grouseberry, 355

Grove, C., 190

Grus americana, 224 canadensis, 12,224

Grus canadensis, Nesting in the Yorkton Wetland Complex, Saskatchewan, Sandhill Crane, 224

Guillemot, Pigeon, 13,53
Gull, Black-headed, 13

Bonaparte's, 13

Glaucous, 13

Glaucous-winged, 13,604

Great Black-backed, 70

Herring, 13,70

Ivory, 13

Mew, 13

Ring-billed, 13

Ross's, 13

Sabine's, 13

Thayer's, 13

Gulo gulo, 18,357,364,566

Gutierrezia sarothrae, 645

Gymnocarpium dryopteris, 650 dryopteris ssp. dryopteris, 279,449 jessoense ssp. parvulum, 450

Gyrfalcon, 7,479

Habenaria hyperborea, 454 obtusata, 454

Hackberry, 218

Haedrich, R.L., 440

Haematopus bachmani, 13

Hairgrass, Mountain, 452

Slender, 452

Hake, Silver, 475

Halecium beanii, 558

curvicaule, 561

halecinum, 558

labrosum, 558

lankesteri, 557

muricatum, 557

scutum, 559

sessile, 559

undulatum, 559

Halenia deflexa, 448

Haliaeetus leucocephalus, 12,601

Haliaeetus leucocephalus, Feeding on Spawning Plainfin Midshipman, Porichthys notatus, at Crescent Beach, British Columbia, Bald Eagles, 601

Hare, Snowshoe, 17,246,306,314,360,591

Harebell, 260

Harrier, Northern, 12,227,479

Harrier, Circus cyaneus, Attacks on Greater Sage-Grouse, Centrocercus urophasianus, in Southern Alberta, Northern, 479

Harris, I.W.E., C.F. Drury, R.R. Simard, and T.Q. Zhang. Density and Richness of Benthic Invertebrate Populations in the North Sydenham River of Southwestern Ontario (1996-2000) Compared with Those of the St. Clair River (1990-1995), 267

Harris, S.A. An Occurrence of the Hawkweed-Leaved Saxifrage, Saxifraga hieraciifolia, in Southern British Columbia, and its Palaeobotanical Implications, 304

Hartlaubella gelatinosa, 561

Hawk, Eastern Red-tailed, 122

Ferruginous, 479

Harlan's, 122

Krider's, 122

Red-tailed, 7,122,179,479

Rough-legged, 12

Sharp-shinned, 12

Swainson's, 12,479 
Hawks in Eastern Kansas, Winter Occurrence of Harlan's, Buteo jamaicensis harlani, and Krider's, B. j. borealis, 122

Hawk's-beard, Annual, 464

Hawkweed, White, 299

Hawthorn, 311

Haxton, T. Movement of Lake Sturgeon, Acipenser fulvescens, in a Natural Reach of the Ottawa River, 541

Heal-all, 297

Heard, D.C. 565

Heather, 354 Pink Mountain, 461

Hebda, A., 475

Hedysarum boreale ssp. mackenzii, 279 boreale ssp. mackenzii f. niveum, 295

Helictotrichon hookeri, 262

Hellebore, White, 288

Helminthoidichnites, 578

Hemidactylium scutatum, 19

Hemlock, 362

Coastal Western, 130

Eastern, 590,622

Interior Western, 130

Mountain, 1,130

Western, 1,215,353,566

Hepatica, Sharp-lobed, 650

Herbertus sp., 214

Heron, Great Blue, 7,603

Heteroscelus incanus, 13

Hickory, 19,622

Bitternut, 218,649

Hieracium albiflorum, 278

aurantiacum, 536

caespitosum, 536

florentinum, 536

pilosella, 536

Hierochloe alpina, 67 alpina ssp. alpina, 279

Hirundo rustica, 15

Histrionicus histrionicus, 12,249

Histrionicus histrionicus, in Greenland, Distribution and Conservation of the Harlequin Duck, 249

Hobson, K.A., 347

Holloran, M.J. and S.H. Anderson. Direct Identification of Northern Sage-grouse, Centrocercus urophasianus, Nest Predators Using Remote Sensing Cameras, 308

Holt, D.W., M.T. Maples, and C. Savok. Black Color Morph of the Brown Lemming, Lemmus trimucronatus $=L$. sibiricus, 466

Hookeria sp., 211

Horehound, Northern Water, 463

Hornwort, 291

Horsetail, 103

Field, 450

Marsh, 450

Variegated, 280,450

Houston, C.S., Reviews by, 326,336,337,338

Huckleberry, Red, 214

Hudson, R.J., 196

Huettmann, F., 53

Huettmann, F., Reviews by, 154,321,325,330,332,334,500, $507,662,664,667$

Hulteniella integrifolia, 66
Hulteniella integrifolia (Asteraceae), in Québec, Eco-geographical and Cytological Notes on the Entire-leaved Daisy, 66

Hummingbird, Anna's, 14 Rufous, 7

Hutchinson, R. and H. Goulet, Review by, 487

Hydractinia carica, 561 minuta, 561 polyclina, 557

Hydrallmania falcata, 557

Hydroids (Cnidaria) of Northumberland Strait, Atlantic Canada, with Observations on Their Life Cycles and Distributions, Subtidal, 555

Hylocomium splendens, 210,535

Hypericum perforatum, 536

Hypogymnia austerodes, 62 imshaugii, 62

occidentalis, 62

physodes, 62,535

tubulosa, 62

Iris setosa ssp. interior, 279

Iris, Wild, 288

Ironwood, 650

Ixoreus naevius, 15

Jackal, Blackbacked, 424

Jackrabbit, White-tailed, 309

Jacob's-ladder, Northern, 462

Tall, 297

Jaeger, Long-tailed, 13

Parasitic, 7

Pomarine, 13

Jay, Blue, 179,193

Gray, 14

Stellar's, 7

John, R., Reviews by, 151,153,320,335,489,659,663,668

Johnston, V., 302

Joyce, T.L., 57

Juglans nigra, 218

Junco hyemalis, 16,179

Junco, Dark-eyed, 16,179

Juncus spp., 103 balticus ssp. alaskanus, 449

balticus var. littoralis, 279

filiformis, 449

triglumis ssp. albescens, 279

Juniper, Common, 213

Ground, 451

Juniperus communis, 211,449

Jutras, J., 377

Kaiser, G., 53

Kalmia microphylla, 211 polifolia, 279

Kamler, J.F., 472

Kamler, J.F. and P.S. Gipson. Space and Habitat Use by Male and Female Raccoons, Procyon lotor, in Kansas, 218

Kamler, J.F. and W.B. Ballard. White Color Phase of the Swift Fox, Vulpes velox, 468

Kamler, J.F., K. Keeler, G. Wiens, C. Richardson, and P.S. Gipson. Feral Dogs, Canis familiaris, Kill Coyote, Canis latrans, 123 
Kansas, Space and Habitat Use by Male and Female Raccoons, Procyon lotor, in, 218

Kansas, Winter Occurrence of Harlan's, Buteo jamaicensis harlani, and Krider's, B. j. borealis, Hawks in Eastern, 122

Karagatzides, J.D., 523

Keeler, K., 123

Kennedy, C.E., 278

Keratosum maximum, 557

Kestrel, 424 American, 12

Killdeer, 7,311

Kindbergia sp., 211

Kingbird, Eastern, 14,169

Kingfisher, Belted, 14

Kinglet, Golden-crowned, 7,177 Ruby-crowned, 15

Kinley, T.A., J. Bergenske, J.-A. Davies and D. Quinn. Characteristics of Early-Winter Caribou, Rangifer tarandus caribou, Feeding Sites in the Southern Purcell Mountains, British Columbia, 352

Kirstein, F., 531

Kittiwake, Black-legged, 13

Knot, Red, 13

Knotweed, Common, 290 Striate, 290

Kobresia hyperborea, 453 myosuroides, 449 siberica, 449 simpliciuscula, 279

Kobresia, Simple, 288

Koeleria cristata, 262 macrantha, 25

Koenigia islandica, 449

Koenigia, Iceland, 455

Kohn, B.E., 419

Koons, D.N. and J.J. Rotella. Have Lesser Scaup, Aythya affinis, Reproductive Rates Declined in Parkland Manitoba?, 582

Labrador, Range Extensions of Logperch, Percina caprodes, and Longnose Dace, Rhinichthys cataractae, in Newfoundland and, 57

Labrador-tea, 461

Lafoea dumosa, 557

fruticosa, 561

gracillima, 557

Lafoeina tenuis, 557

Lagopus spp., 365

lagopus, 12,480

leucurus, 12

mutus, 12

Lamb, E.G. and W. Megill. The Shoreline Fringe Forest and Adjacent Peatlands of the Southern Central British Columbia Coast, 209

Lamprey, Sea, 59

Lane, R.K. and M. Pearman. Comparison of Spring Return Dates of Mountain Bluebirds, Sialia currucoides, and Tree Swallows, Tachycineta bicolor with Monthly Air Temperatures, 110

Lanius excubitor, 14

Laomedea neglecta, 559

Lapointe, S., L. Bélanger, J.-F. Giroux, B. Filion. Effects of Plant Cover Improvements for Nesting Ducks on Grassland Songbirds, 167
Lappula occidentalis, 279

Larch, 436

Alpine, 353

Larivière, S., 347

Larivière, S., Review by, 504

Larix laricina, 436,590 lyalli, 353

Lark, Horned, 15,169

Larkspur, Tall, 292

Larus argentatus, 13,70

canus, 13

delawarensis, 13

glaucescens, 13,604

hyperboreus, 13

marinus, 70

philadelphia, 13

ridibundus, 13

thayeri, 13

Lasionycteris noctivigans, 652

Lasiurus borealis, 651 cinereus, 652

Lasiurus borealis, from Cypress Hills Provincial Park, Saskatchewan: A Response to Climate Change?, New Records of the Eastern Red Bat, 651

Lathyrus sativus, 198

Lauff, R., Review by, 488

Laurel, Bog, 213

Western Bog, 214

Leather-leaf, 436,461

Ledum groenlandicum, 211,449

Leech, 380

Leek, Wild, 650

Lein, M.R., 76

Lemming, Brown, 7,466

Northern Bog, 17

Norwegian, 467

Siberian, 17

Lemming, Lemmus trimucronatus $=$ L. sibiricus, Black Color Morph of the Brown, 466

Lemmus lemmus, 467

sibiricus, 17,466

trimucronatus, 466

Lemmus sibiricus, Black Color Morph of the Brown Lemming, Lemmus trimucronatus, 466

Lemmus trimucronatus $=$ L. sibiricus, Black Color Morph of the Brown Lemming, 466

Lemons, P.R., W.B. Ballard, R.M. Sullivan, and M.A. Sovada. Den Site Activity Patterns of Adult Male and Female Swift Foxes, Vulpes velox, in Northwestern Texas, 424

Leonard, T.D., 477

Leopardbane, Snow, 299

Lepeophtheirus salmonis, 634

Lepeophtheirus salmonis, Infestation on Juvenile Pink Salmon, Oncorhynchus gorbuscha, in Nearshore Habitat, First Report of a Sea Louse, 634

Lepidium sativum, 280

Lepidozia reptans, 535

Leptarrhena pyrolifolia, 279

Leptogium saturminum, 62

Lepus americanus, 17,246,306,314,360,591 townsendii, 309

Lesquerella arctica ssp. arctica, 279 calderi, 279

Leucanthemum integrifolium, 66 
Leuckartiara octona, 561

Leucosticte tephrocotis, 16

Lice, Sea, 636

Lichen, 61

$$
\text { Hair, 39,44 }
$$

Horsehair, 352

Lichens (Bryoria) in High-Elevation Oldgrowth Conifer Forests, On the Dispersal of Hair, 44

Lichens (Bryoria) in the Canopies of High-elevation Oldgrowth Conifer Forests, On the Vertical Zonation of Hair, 39

Lichens in Well-ventilated Stands in East-central British Columbia, Notes on the Populus "Dripzone Effect" on, 61

Lily, Alp, 288

Glacier, 304

Lily-of-the-valley, False, 214,288

Lim, B.K., Reviews by, 487,672

Limnodromus griseus, 13 scolopaceus, 13

Limosa fedoa, 13

haemastica, 13

lapponica, 13

Linard, C., 605

Linaria vulgaris, 280

Lindgren, C.J. A Brief History of Purple Loosestrife, Lythrum salicaria, in Manitoba and its Status in 2001, 100

Lindquist, E.S., C.F. Aquadro, D. McClearn, and K.J. McGowan. Field Identification of the Mice Peromyscus leucopus noveboracensis and P. maniculatus gracilis in Central New York, 184

Line, J.M., 448

Lingonberry, 213

Linnaea borealis, 211,352

borealis var. americana, 449

Listera, 210

Liverwort, 214

Leafy, 214

Lloydia serotina, 279

Lobaria hallii, 64 pulmonaria, 61,352

Lobarion, 63

Lobelia dortmanna, 449

Lobelia, Water, 463

Locoweed, Blackish, 295 Yellow, 260

Locust, Honey, 218

Logperch, 57

Logperch, Percina caprodes, and Longnose Dace, Rhinichthys cataractae, in Newfoundland and Labrador, Range Extensions of, 57

Lomatogonium rotatum ssp. rotatum, 449

Longspur, Lapland, 7 Smith's, 7

Lontra canadensis, 35,380

Loon, Common, 11,254

Pacific, 11

Red-throated, 7

Yellow-billed, 11

Loosestrife, Purple, 100

Loosestrife, Lythrum salicaria, in Manitoba and its Status in 2001, A Brief History of Purple, 100

Lophodytes cucullatus, 12

Lota lota, 59

Lotus corniculatus, 278
Lougheed, C., 53

Lougheed, L., 53

Louse, Sea, 634

Louse, Lepeophtheirus salmonis, Infestation on Juvenile Pink Salmon, Oncorhynchus gorbuscha, in Nearshore Habitat, First Report of a Sea, 634

Lousewort, Woolly, 298,463

Lovegrass, Sand, 645

Loxia curvirostra, 16 leucoptera, 16

Lumpsucker, 254

Lungwort, 352

Drummond's, 462

Lutra canadensis, 18,115

Lutra canadensis, in a Highly Urbanized Area, Incidence of Mink, Mustela vison, and River Otter, 115

Lycopodium alpinum, 279

clavatum, 214

clavatum var. monostachyon, 279,449

complanatum, 279,449

Lycopus americanus, 103

uniflorus, 449

Lynx canadensis, 360

canadensis canadensis, 360

canadensis subsolanus, 360

(Felis) lynx, 360

lynx, 18

rufus, 193,361,472

Lynx, 18

Canada, 360

Eurasian, 360

Lynx canadensis, in Canada*, A Review of the Canada Lynx, 360

Lynx, Lynx canadensis, in Canada*, A Review of the Canada, 360

Lynx rufus, and Raccoons, Procyon lotor, Relative to Home Range

Boundaries, Capture Locations of Coyotes, Canis latrans, Bobcats, 472

Lysimachia thyrsiflora, 103

Lythrum spp., 106 salicaria, 100

Lythrum salicaria, in Manitoba and its Status in 2001, A Brief History of Purple Loosestrife, 100

Maass, W., 531

MacDonald, S.D., 140

MacNaughton, R.B. Planispiral Burrows from a Recent Lacustrine Beach, Gander Lake, Newfoundland, 577

MacPherson, A., Review by, 666

Madrepora oculata, 440

Magpie, Black-billed, 15,30,308

Mahoney, S.P., 236

Maianthemum canadense, 535

dilatatum, 211,280

racemosum, 650

trifolium, 449

Malaxis brachypoda, 302 monophyllos var. brachypoda, 302 monophyllos var. monophyllos, 302

Malaxis, One-leaved, 302

Malaxis monophyllos var. brachypoda, One-leaved Malaxis, new to the Northwest Territories, Canada, 302

Malaxis, new to the Northwest Territories, Canada, Malaxis monophyllos var. brachypoda, One-leaved, 302 
Mallard, 11,254

Mallotus villosus, 70

Maltais, J., 399

Malus fusca, 213

Manitoba and its Status in 2001, A Brief History of Purple Loosestrife, Lythrum salicaria, in, 100

Manitoba, First record of the Deepwater Sculpin, Myoxocephalus thompsonii, from George Lake in Whiteshell Provincial Park, 642

Manitoba?, Have Lesser Scaup, Aythya affinis, Reproductive Rates Declined in Parkland, 582

Manitoba, Sympatric Presence of Low and High Gillraker Forms of Cisco, Coregonus artedi, in Lake Athapapuskow, 49

Maple, 378

Black, 649

Red, $185,436,469,532,590,622$

Silver, 470

Sugar, 389,532,590,622,649

Maples, M.T., 466

Marine Turtle Newsletter, 166,344,513,678

Marmot, Alaskan, 7 Hoary, 17

Marmota broweri, 17 caligata, 17 monax, 17,247,591

Marsh-marigold, 456 Floating, 456

Marten, 246,591 American, 18,35,190

Marten, Martes americana, Reintroduction in Vermont, Assessing an American, 190

Martes americana, 18,35,190,246,591 pennanti, 193,591

Martes americana, Reintroduction in Vermont, Assessing an American Marten, 190

Masse, D., 377

Matricaria perforata, 280

Mawhinney, K., 236

Mayweed, Scentless, 299

McCarthy, J., Review by, 502

McClearn, D., 184

McDonald, J.E. Jr. Methods for Capturing Free-Ranging Black Bears, Ursus americanus, in Difficult Locations, 621

McFarlane-Tranquilla, L., F. Huettmann, C. Lougheed, L. Lougheed, N. Parker, and G. Kaiser. Sightings of Vagrant Pacific Alcids in Desolation Sound, British Columbia, 53

McGowan, K.J., 184

McManus, R., Jr., 611

McNicholl, M.K., Review by, 505

McPherson, M., 82

Meadow-foxtail, Water, 282

Meadowlark, Eastern, 169,177 Western, 16

Meadowrue, Alpine, 292 Few-flowered, 292

Mech, L.D. Incidence of Mink, Mustela vison, and River Otter, Lutra canadensis, in a Highly Urbanized Area, 115

Medicago sativa ssp. falcata, 448

Megill, W., 209

Melandrium taimyrense, 456

Melanelia subaurifera, 62
Melanerpes formicivorus, 311

Melanitta fusca, 12

nigra, 12

perspicillata, 12

Meleagris gallopavo, sspp. 609,645 gallopavo intermedia, 645

Meleagris gallopavo intermedia, interactions, Coyote, Canis latrans - Rio Grand Turkey, 645

Meles meles, 243

Melic, False, 285

Melilotus alba, 450 officinalis, 168

Melospiza lincolnii, 16,169 melodia, 16,169,179

Mentha arvensis, 103

Menyanthes trifoliata, 279,436,449

Menziesia ferruginea, 214

Mephitis mephitis, 30,227,348,380

Mercenaria mercenaria, 555

Merganser, Common, 12

Hooded, 7

Red-breasted, 12

Mergus merganser, 12 serrator, 12

Merlin, 12

Merluccius bilinearis, 475

Mertensia drummondii, 449

Mice Peromyscus leucopus noveboracensis and P. maniculatus gracilis in Central New York, Field Identification of the, 184

Microseris borealis, 214

Microtus spp., 245,348,466

chrotorrhinus, 246

longicaudus, 17

miurus, 17

oеconomus, 17

pennsylvanicus, $17,246,466$

xanthognathus, 17

Middleton, J., 393

Midshipman, Plainfin, 601

Midshipman, Porichthys notatus, at Crescent Beach, British Columbia, Bald Eagles, Haliaeetus leucocephalus, Feeding on Spawning Plainfin, 601

Milk-vetch, Indian, 459

Bodin's, 295

Chick-pea, 295

Pulse, 295

Mimulus guttatus, 279

Mink, 115,227,586,591 American, 18,35,348

Mink, Mustela vison, and River Otter, Lutra canadensis, in a Highly Urbanized Area, Incidence of, 115

Mink, Mustela vison, Populations under Varying Harvest Regimes, Age Structure Differences in American, 35

Minuartia dawsonensis, 279

elegans, 279

obtusiloba, 279

rossii, 449

rubella, 449

yukonensis, 279

Miramichi estuary, 562

Mistletoe, 306

Mitella spp., 352

diphylla, 650

Mitrewort, 352 
Molothrus ater, 16,348

Monkeyflower, Yellow, 298

Monkshood, Mountain, 291

Monobrachium parasiticum, 562 parasitum, 562

Montgomery, K., Review by, 505

Moonwort, 450

Moose, 7,316,357,400,547,591

Alaskan, 7

Morton, A.B. and R. Williams. First Report of a Sea Louse, Lepeophtheirus salmonis, Infestation on Juvenile Pink Salmon, Oncorhynchus gorbuscha, in Nearshore Habitat, 634

Morus rubra, 218

Moruzzi, T.L., K.J. Royar, C. Grove, R.T. Brooks, C. Bernier, F.L. Thompson, Jr., R.M. DeGraaf, and T.K. Fuller. Assessing an American Marten, Martes americana, Reintroduction in Vermont, 190

Moschatel, 298,463

Moss, 395

Motacilla alba, 15 lugens, 15

Mouse, 365,380

Deer, $7,184,246,390$

Field, 245

Meadow Jumping, 17

Northwestern Deer, 7,17

Prairie Deer, 185

White-footed, 184,309

Woodland Jumping, 246

Mugwort, Aleutian, 463

Mulberry, Red, 218

Mulinia lateralis, 555

Murray, L., M.H. Papst, and J.D. Reist. First record of the Deepwater Sculpin, Myoxocephalus thompsonii, from George Lake in Whiteshell Provincial Park, Manitoba, 642

Murre, Common, 13

Murrelet, Ancient, 13

Kittlitz's, 13

Marbled, 13,53

Mushroom, 380

Muskrat, 17,115,365,380,590

Mustard, Wormseed, 294

Mustela sp., 247

erminea, $18,193,245$

frenata, 193,246,313

nivalis, 18,245

vison, $18,35,115,227,348,586,591$

Mustela erminea, in a Forested Landscape, Food Habits of Ermine, 245

Mustela frenata, Hunting Behavior in Central West Virginia, Observations of Long-tailed Weasel, 313

Mustela vison, and River Otter, Lutra canadensis, in a Highly Urbanized Area, Incidence of Mink, 115

Mustela vison, Populations under Varying Harvest Regimes, Age Structure Differences in American Mink, 35

Myadestes townsendi, 15

Myotis lucifugus, 17,652

Myoxocephalus quadricornis, 643 thompsonii, 642

Myoxocephalus thompsonii, from George Lake in Whiteshell Provincial Park, Manitoba, First record of the Deepwater Sculpin, 642

Myrica gale, 211
Napaeozapus insignis, 246

National Recovery Strategy for Species at Risk in the Sydenham River: A Ecosystem Approach, 678

Needle-and-Thread, 25,285

Neotoma cinerea, 17

Nephroma resupinatum, 62

New Brunswick, Comparison of the Ground Vegetation in Spruce Plantations and Natural Forest in the Greater Fundy Ecosystem, 531

Newfoundland and Labrador, Range Extensions of Logperch, Percina caprodes, and Longnose Dace, Rhinichthys cataractae, in, 57

Newfoundland and Mainland Populations of North American Black Bears, Ursus americanus, Phenotypic Variation in Skull Size and Shape Between, 236

Newfoundland, First Record of Mink Frog, Rana septentrionalis, from Insular, 477

Newfoundland, Planispiral Burrows from a Recent Lacustrine Beach, Gander Lake, 577

Newt, Red-spotted, 19

New York, Field Identification of the Mice Peromyscus leucopus noveboracensis and $P$. maniculatus gracilis in Central, 184

Nighthawk, Common, 7

Nightshade, Enchanter's, 296

Nodobryoria, 39,44

abbreviata, 45

oregana, 39,45

Norment, C.J. Patterns of Nestling Feeding in Harris's Sparrows, Zonotrichia querula and White-crowned Sparrows, Z. leucophrys, in the Northwest Territories, Canada, 203

North America, Status of the Largest Breeding Concentration of Atlantic Puffins, Fratercula arctica, in, 70

North Carolina, Marsh Rice Rat, Oryzomys palustris, Predation on Forster's Tern, Sterna forsteri, Eggs in Coastal, 654

Northwest Territories and Nunavut, Canada, II, Additions and Range Extensions to the Vascular Plant Flora of the Continental, 448

Northwest Territories, Canada, Malaxis monophyllos var. brachypoda, One-leaved Malaxis, new to the, 302

Northwest Territories, Canada, Patterns of Nestling Feeding in Harris's Sparrows, Zonotrichia querula and Whitecrowned Sparrows, Z. leucophrys, in the, 203

Notophthalmus viridescens, 19

Nova Scotia, The Wood Turtle, Glyptemys insculpta, at River Denys: A Second Population for Cape Breton Island, 415

Nowellia curvifolia, 535

Nucifraga columbiana, 15

Numenius phaeopus, 13 tahitiensis, 13

Nunavut, Canada, II, Additions and Range Extensions to the Vascular Plant Flora of the Continental Northwest Territories and, 448

Nutcracker, Clark's, 15

Nuthatch, Red-breasted, 7,177 White-breasted, 177

Nutsedge, Purple, 106 Yellow, 106

Nyctea scandiaca, 14

Oak, 19,394

Bur, 218 
Chinkapin, 218

Northern Red, 622

Red, 590

Oak-fern, Nahanni, 451

Obelia geniculata, 559

longissima, 557

Oceanodroma furcata, 11

leucorhoa, 11

Ochotona collaris, 17

Octopus bimaculatus, 117

briareus, 117

cyanea, 117

vulgaris, 117

Octopus, Giant Pacific, 117

Octopus, Enteroctopus dofleini (Wülker, 1910), An in situ Observation of Webover Hunting by the Giant Pacific, 117

Odocoileus hemionus, 18,309,316,357,657 virginianus, 309,316,357,389,407,567,590

Odocoileus hemionus, in Central Colorado, Aggressive Interactions of Rocky Mountain Elk, Cervus elaphus nelsoni, During the Calving Season Toward Mule Deer, 316

Odontoschisma denudatum, 535

Oenanthe oenanthe, 15

Ogden, L.E., Review by, 503

Okello, M.M., 196

Oncorhynchus gorbuscha, 59,634

keta, 638

kisutch, 639

nerka, 603,634

tshawytscha, 639

Oncorhynchus gorbuscha, in Nearshore Habitat, First Report of a Sea Louse, Lepeophtheirus salmonis, Infestation on Juvenile Pink Salmon, 634

Ondatra zibethicus, 17,115,365,380,590

Onobrychis viciifolia, 280

Ontario, Canopy Interception of Acid Deposition in Southern, 523

Ontario, Lack of Evidence for Impact of the European White Birch, Betula pendula, on the Hydrology of Wainfleet Bog, 393

Ontario, Landscape Influence on Canis Morphological and Ecological Variation in a Coyote-Wolf C. lupus $\times$ latrans Hybrid Zone, Southeastern, 589

Ontario (1996-2000) Compared with Those of the St. Clair River (1990-1995), Density and Richness of Benthic Invertebrate Populations in the North Sydenham River of Southwestern, 267

Ontario, Winter Bird Use of Urban and Rural Habitats in, 173

Opercularella lacerata, 558 pumila, 557

Oporornis tolmiei, 15

Orchid, Northern Bog, 454

Northern Green, 454

Oreamnos americanus, 18,565

Oreamnos americanus, in East-central British Columbia, Seasonal Habitat Use and Movements of Mountain Goats, 565

Oregonia gracilis, 117

Oritsland, N.A., 430

Orobanche fasciculata, 279

Orthopyxis integra, 557

Oryctolagus cuniculus, 242

Oryzomys palustris, 654
Oryzomys palustris, Predation on Forster's Tern, Sterna forsteri, Eggs in Coastal North Carolina, Marsh Rice Rat, 654

Osmerus mordax, 59

Osmorhiza depauperata, 279,449

Osprey, 12

Ostrea excavata, 440

Ostrya virginiana, 650

Otisorex, 82

Ottawa Field-Naturalists' Club Awards for 2002, The, 679

Ottawa Field-Naturalists' Club 14 January 2003, Minutes of Otter, 380 the $124^{\text {th }}$ Annual Business Meeting of The, 514

River, $18,35,115$

Sea, 3

Otter, Lutra canadensis, in a Highly Urbanized Area, Incidence of Mink, Mustela vison, and River, 115

Otus kennicottii, 14

Ouellet, J.-P., 399,546

Ovalipes ocellatus, 555

Ovis dalli, 18 dalli dalli, 7

Owl, Boreal, 14

Great Gray, 14

Great Horned, 14,227,479

Northern Hawk, 14,306

Saw-whet, 14

Short-eared, 14,466

Snowy, 14

Owl, Surnia ulula, Nest on a Man-made Structure in Alaska, A Northern Hawk, 306

Oxalis montana, 535

Oxycoccus microcarpus, 449 oxycoccos, 213

Oxylebius pictus, 118

Oxytropis arctica, 449

Oxytropis campestris, 260

Oxytropis campestris $\mathrm{ssp}$. roaldii, 279

Oxytropis deflexa, 67

Oxytropis deflexa ssp. foliolosa, 449,460

Oxytropis nigrescens ssp. nigrescens, 279

Oxyura jamaicensis, 12

Oystercatcher, Black, 13

Pachistima myrsinites, 352

Pagophila eburnea, 13

Paintbrush, Port Clarence, 298

Pandion haliaetus, 12

Panicum virgatum, 218

Panther, Florida, 113

Papaver keelei, 457 macounii ssp. discolor, 449

Papst, M.H., 642

Paraonis fulgens, 578

Parelaphostrongylus tenuis, 407

Parker, N., 53

Parmelia hygrophila, 62 sulcata, 62

Parmeliopsis ambigua, 62 hyperopta, 62

Parnassia fimbriata, 279 kotzebuei, 449 palustris var. neogaea, 449

Parus major, 424

Passer domesticus, 177 
Passerculus sandwichensis, 16

Passerella iliaca, 16

Peanut, Hog, 650

Pearman, M., 110

Pedicularis flammea, 449

lanata, 279,449

macrodonta, 449

parviflora, 463

Peltigera spp., 352

Penguin, Emperor, 231,430

King, 231

Percina caprodes, 57

Percina caprodes, and Longnose Dace, Rhinichthys cataractae, in Newfoundland and Labrador, Range Extensions of Logperch, 57

Pericladium mirabile, 560

Perigonimus sessilis, 561

Perisoreus canadensis, 14

Peromyscus spp., 193,309,348

keeni, 17

leucopus, 184

leucopus noveboracensis, 184

maniculatus, 17,184,246,390

maniculatus bairdii, 184

maniculatus gracilis, 184

maniculatus nubiterrae, 184

Peromyscus leucopus noveboracensis and P. maniculatus gracilis in Central New York, Field Identification of the Mice, 184

Peromyscus maniculatus gracilis in Central New York, Field Identification of the Mice Peromyscus leucopus noveboracensis and, 184

Perry, R. and T.L. Joyce. Range Extensions of Logperch, Percina caprodes, and Longnose Dace, Rhinichthys cataractae, in Newfoundland and Labrador, 57

Persicaria vivipara, 67

Petasites frigidus ssp. palmatus, 449 palmatus, 464 sagitatus, 449

Peterson, A.P., Review by, 337

Petrochelidon pyrrhonota, 15

Petromyzon marinus, 59

Phalacrocorax auritus, 11 pelagicus, 11

Phalaris arundinacea, 103,168,279,449

Phalarope, Red, 13

Red-necked, 7

Wilson's, 169

Phalaropus fulicarius, 13

lobatus, 13

tricolor, 169

Phasianus colchicus, 479

Pheasant, Ring-necked, 479

Phenacomys ungava, 17

Phillips, G.E., 316

Phleum pratense, 168,198,280,536

Phlox hoodii, 262,279

Phlox, Moss, 297

Phoca groenlandica, 121 hispida, 121,433

Phoebastria nigripes, 11

Phoebe, Eastern, 14 Say's, 14

Phragmites australis, 103

Phryma leptostachya, 650
Phyllodoce coerulea, 449

empetriformis, 354,449

glanduliflora, 354

Phylloscopus borealis, 15

Physcia aipolia, 62

Pica hudsonia, 15 pica, 30

Picea spp., 61,203,306,362,394

engelmannii, 40,44,353

engelmannii $\mathrm{x}$ glauca, 61

glauca, 1,76,130,307,353,378,532,590

glauca X engelmannii, 566

mariana, 1,72,130,362,436,532,590

rubens, 532

sitchensis, 1,212

Picoides arcticus, 14

pubescens, 14,179

tridactylus, 14

villosus, 14, 177

Pigeon, Band-tailed, 14

Pigmyweed, 458

Pika, Collared, 7

Pike, Northern, 59

Pin-Cherry, 538

Pine, 19,362,394

Eastern White, 590,622

Jack, 91,130

Limber, 91

Lodgepole, 76,91,281,353,362,566

Ponderosa, 91,628

Red, 590

Shore, 213

Whitebark, 353,566

Pinicola enucleator, 16

Pintail, Northern, 12,605

Pintails, Anas acuta, Initiate Rapid Follicular Growth During Spring Migration?, Do Female Northern, 605

Pinus spp., 19,362,394

albicaulis, 353,566

banksiana, 91,130

contorta, 61,76,91,211,353,362,566

contorta ssp. latifolia, 279

flexilis, 91

ponderosa, 91,628

resinosa, 590

strobus, 590

Pipit, American, 15

Piranga ludoviciana, 16

Placobdella sp., 380

Plagiothecium sp., 211

Plangtichnus, 578

Planolites, 578

Plantago major, 280

Plantain, Common, 298

Platanthera aquilonis, 449 hyperborea, 454 obtusata, 449

Platanus occidentalis, 646

Platismatia glauca, 62

Plectrophenax nivalis, 16,177

Plethodon cinereus, 22

glutinosus, 19

hoffmani, 19

Pleurozium schreberi, 535

Plover, Black-bellied, 12 
Semipalmated, 12

Pluvialis dominica, 12

fulva, 12

squatarola, 12

Poa arctica, 67,449

compressa, 280,536

leptocoma, 279

porsildii, 279

pratense, 536

pratensis, 168,196,258

pratensis ssp. alpigena, 279

secunda ssp. secunda, 279

trivialis, 280

Podiceps auritus, 11

grisegena, 11

Podilymbus podiceps, 11

Poecile atricapillus, 15,179

cincta, 15

gambeli, 15,76

hudsonica, 15

rufescens, 15

Poecile gambeli, to Playback of Different Song Types, Response of Male Mountain Chickadees, 76

Point Pelee Natural History News, 167,343,513,677

Polargrass, 452

Polemonium acutiflorum, 279

boreale, 449

boreale forma albiflorum, 448

Polygonum spp., 103

achoreum, 280

aviculare, 280

caurianum ssp. hudsonianum, 455

fowleri, 448

hudsonianum, 449

hydropiper, 536

ramosissimum, 448

Polytrichastrum alpinum, 66

Polytrichum spp., 536

commune, 535

formosum, 537

piliferum, 66

strictum, 395

Pondweed, Fine-leaved, 281

Giant, 281,451

Ponomarenko, S., 125

Poole, K.G. A Review of the Canada Lynx, Lynx canadensis, in Canada*, 360

Poole, K.G. and D.C. Heard. Seasonal Habitat Use and Movements of Mountain Goats, Oreamnos americanus, in East-central British Columbia, 565

Poplar, Balsam, 196

Populus, 61 alba, 536

balsamifera, 196,582

deltoides, 645

grandidentata, 436

tremuloides, 61,76,91,196,362,378,395,436,582,590,652 trichocarpa, 61

Populus "Dripzone Effect" on Lichens in Well-ventilated Stands in East-central British Columbia, Notes on the, 61

Porcupine, 469

American, 17

Porcupine, Erethizon dorsatum, Dens in Wisconsin, Death of Gray Wolves, Canis lupus, in, 469
Porichthys notatus, 601

Porichthys notatus, at Crescent Beach, British Columbia, Bald Eagles, Haliaeetus leucocephalus, Feeding on Spawning Plainfin Midshipman, 601

Porzana carolina, 12

Potamogeton spp., 273

filiformis var. borealis, 279

natans, 449

obtusifolius, 449

porsildiorum, 451

richardsonii, 449

vaginatus, 279,449

Potentilla anserina, 103

arguta, 260

hyparctica, 449

nivea, 279,449

porsildiorum, 449

recta, 536

tridentata, 449

Powell, K.G., 477

Primrose, Greenland, 296 Mealy, 462

Primula egaliksensis, 279 incana, 449

Procyon lotor, 30,193,218,227,348,380,472,591

Procyon lotor, in Kansas, Space and Habitat Use by Male and Female Raccoons, 218

Procyon lotor, Relative to Home Range Boundaries, Capture Locations of Coyotes, Canis latrans, Bobcats, Lynx rufus, and Raccoons, 472

Pronghorn, 309

Prosopium cylindraceum, 59

Prunella vulgaris ssp. lanceolata, 278

Prunus pensylvanica, 538 serotina, 19,395

virginiana, 417

Psathyrostachys juncea, 283

Pseudacris triseriata, 477

Pseudevernion, 63

Pseudocyphellaria anomala, 61

Pseudotriton ruber, 19

Pseudotsuga menziesii, 45,61,91,353,362,628

Ptarmigan, 365

Rock, 12

White-tailed, 12

Willow, 12

Pteridium aquilinum, 214

Ptilidium ciliare, 66

Ptychoramphus aleuticus, 13

Puccinellia deschampsioides, 279

distans, 280

nuttalliana, 280

Puffin, Atlantic, 70

Horned, 14

Tufted, 14

Puffins, Fratercula arctica, in North America, Status of the Largest Breeding Concentration of Atlantic, 70

Puffinus carneipes, 11 griseus, 11 tenuirostris, 11

Puma concolor, 566 concolor coryi, 113

Pungitius pungitius, 59

Purshia tridentata, 628

Pyrola minor, 280,449 
Quail, Japanese, 348

Québec, Eco-geographical and Cytological Notes on the Entire-leaved Daisy, Hulteniella integrifolia (Asteraceae), in, 66

Québec, Ecological Aspects of a Wood Turtle, Glyptemys insculpta, Population at the Northern Limit of its Range in, 377

Quebec, Historical Changes and Current Distribution of Caribou, Rangifer tarandus, in, 399

Quercus spp., 19,394

macrocarpa, 218

muhlenbergii, 218

rubra, 590,622

Quinn, D., 352

Rabbit, European, 242

Rabbitbrush, 645

Raccoon, 30,193,218,227,348,380,472,591

Raccoons, Procyon lotor, in Kansas, Space and Habitat Use by Male and Female, 218

Raccoons, Procyon lotor, Relative to Home Range Boundaries, Capture Locations of Coyotes, Canis latrans, Bobcats, Lynx rufus, and, 472

Racomitrium sp., 213

Ragweed, Mount Sheldon, 299 Western, 645

Ragwort, Tundra, 300

Ramalina thrausta, 62

Rana clamitans, 477

pipiens, 477

septentrionalis, 477

sylvatica, 477

Rana septentrionalis, from Insular Newfoundland, First Record of Mink Frog, 477

Rangifer tarandus, 18,120,253,399

tarandus caribou, 7,39,44,352,546

tarandus granti, 7 tarandus platyrhynchus, 433

Rangifer tarandus caribou, An Aerial Survey Technique for the Forest-Dwelling Ecotype of Woodland Caribou, 546

Rangifer tarandus caribou, Feeding Sites in the Southern Purcell Mountains, British Columbia, Characteristics of Early-Winter Caribou, 352

Rangifer tarandus, in Quebec, Historical Changes and Current Distribution of Caribou, 399

Ranunculus acris, 536

aquatilis var. eradicatus, 280

aquatilis var. hispidulus, 450

eschscholtzii, 280

flammula, 280,449

flammula var. filiformis, 449

nivalis, 449

pallasii, 449

pedatifidus ssp. affinis, 280

pygmaeus, 449

sulphureus, 449

trichophyllus var. hipidulus, 456

Raspberry, 538

Creeping, 295

Dwarf, 295

Rat, Marsh Rice, 654

Rat, Oryzomys palustris, Predation on Forster's Tern, Sterna forsteri, Eggs in Coastal North Carolina, Marsh Rice, 654
Rattlesnake, Massasauga, 393

Raven, Common, 15,30,227,308

Reading, K.L., 448

Reakoff, J.L., 306

Reakoff, M.L., J.L. Reakoff and T. Craig. A Northern Hawk Owl, Surnia ulula, Nest on a Man-made Structure in Alaska, 306

Recovery: An Endangered Species Newsletter, 166,344,678

Recovery Strategy for Bowhead Whales in Canadian Eastern Arctic, 678

Redcedar, Western, 353,566

Redhead, 7

Redpoll, 611

Common, 7

Hoary, 16

Redpoll, Carduelis sp., Winter Visitations in Atlantic Canada, Supposed Periodicity of, 611

Redstart, American, 7,206

Redtop, 168 Spike, 281

Reedgrass, Northern, 282

Purple, 282

Reeves, H.M., Review by, 661

Regehr, H.M., 70

Regulus calendula, 15 satrapa, 15,177

Reindeer, Svalbard, 433

Reist, J.D., 642

Rhinichthys cataractae, 57

Rhinichthys cataractae, in Newfoundland and Labrador, Range Extensions of Logperch, Percina caprodes, and Longnose Dace, 57

Rhizocaulus verticillatus, 557

Rhizomnium sp., 214

Rhodostethia rosea, 13

Rhytidiadelphus sp., 211

Richardson, C., 123

Riparia riparia, 15

Ripley, E.A., 257

Rissa tridactyla, 13

Rithropanopeus harrisii, 555

Robards, M.D., 119

Robichaud, J., 82

Robin, American, 15,177,311

Robin, Turdus migratorius, Autumn and Winter Breeding Records for the American, 311

Rockcress, Boivin's, 292

Cody's, 292

Holboell's, 292

Lyre-leaved, 292

Rockfish, Copper, 118 Puget Sound, 118

Rodway, M.S., H.M. Regehr, and J.W. Chardine. Status of the Largest Breeding Concentration of Atlantic Puffins, Fratercula arctica, in North America, 70

Romo, J.T. Reintroducing Fire for Conservation of Fescue Prairie Association Remnants in the Northern Great Plains, 89

Rosa spp., 258

woodsii, 280

Rose, 258

Western, 295

Rosemary, Bog, 296

Rosy-Finch, Gray-crowned, 16

Rotella, J.J., 582 
Royar, K.J., 190

Rubus spp., 72,533,594

acaulus, 459

arcticus ssp. acaulis, 449

pedatus, 211,280

pubescens, 280

strigosus, 535

Rumex acetosa ssp. alpestris, 280

acetosella, 536

crispus, 536

occidentalis, 103

Rush, Baltic, 288

Dwarf Scouring, 450

Spike, 103

Whitish, 288

Rye, Canada Wild, 452

Russian Wild, 283

Thick-spike Wild, 283

Sage, 25

Prairie, 258

Silver, 25

Sage-Grouse, Greater, 25,479

Northern, 308

Sage-Grouse, Centrocercus urophasianus, in Canada, Distribution, Abundance, and Status of the Greater, 25

Sage-Grouse, Centrocercus urophasianus, in Southern Alberta, Northern Harrier, Circus cyaneus, Attacks on Greater, 479

Sage-grouse, Centrocercus urophasianus, Nest Predators Using Remote Sensing Cameras, Direct Identification of Northern, 308

Sagebrush, 25,645

Sagittaria spp., 168

Saher, D.J., 479

Salal, 213

Salamander, Four-toed, 19

Jefferson, 19

Mountain Dusky, 19

Northern Red, 19

Redback, 19

Ridge, 19

Slimy, 19

Valley, 19

Woodland, 19

Salamanders Along Forest-Farmland Edges, Distribution of, and Microhabitat Use by, Woodland, 19

Salix spp., 103,203

alaxensis ssp. longistylis, 280

arbusculoides, 280

arctica, 66,280

arctophila, 280

athabascensis, 449

brachycarpa ssp. niphoclada, 289

commutata, 280

fuscescens, 280

hastata, 280

herbacea, 67

lanata ssp. richardsonii, 289

lutea, 449

monticola, 289

niphoclada, 280

polaris, 280,449

polaris ssp. pseudopolaris, 454

pseudomonticola, 280 reticulata, 67

richardsonii, 280

rotundifolia $\mathrm{ssp}$. rotundifolia, 280,448

Salmo salar, 59,634

trutta, 634

Salmon, Atlantic, 59,634

Kokanee, 603

Pink, 59,634

Sockeye, 634

Salmon, Oncorhynchus gorbuscha, in Nearshore Habitat, First Report of a Sea Louse, Lepeophtheirus salmonis, Infestation on Juvenile Pink, 634

Salvelinus alpinus, 59,254,634

fontinalis, 59

namaycush, 59,642

Sambucus racemosa, 538

Sander vitreus, 542

Sander-Regier, R., Reviews by, 157,158,159,327,339,497,498

Sanderling, 13

Sandpiper, Baird's, 13

Buff-breasted, 13

Least, 7

Pectoral, 13

Rock, 13

Semipalmated, 13

Solitary, 13

Spotted, 13

Upland, 13

Western, 13

Sandreed, Prairie, 645

Sandwort, Alpine, 291

Boreal, 456

Northern, 290

Rock, 290

Sanguisorba canadensis ssp. latifolia, 280 officinalis, 213

Sapsucker, Red-breasted, 7 Yellow-bellied, 7

Sarsia tubulosa, 561

Saskatchewan: A Response to Climate Change?, New Records of the Eastern Red Bat, Lasiurus borealis, from Cypress Hills Provincial Park, 651

Saskatchewan, Effects of Season of Burning on the Microenvironment of Fescue Prairie in Central, 257

Saskatchewan, Extralimital Sighting of a Polar Bear, Ursus maritimus, in northeast, 648

Saskatchewan, Sandhill Crane, Grus canadensis, Nesting in the Yorkton Wetland Complex, 224

Savok, C., 466

Saxifraga aizoides, 67

caespitosa ssp. uniflora, 449

cеrnua, 449

foliolosa, 449

hieracifolia, 304,449

hirculus, 449

nelsoniana ssp. porsildiana, 449

nivalis, 449

oppositifolia, 67

punctata ssp. porsildiana, 459

Saxifraga hieraciifolia, in Southern British Columbia, and its Palaeobotanical Implications, An Occurrence of the Hawkweed-Leaved Saxifrage, 304

Saxifrage, Alpine, 459

Hawkweed-Leaved, 304

Leather-leaved, 294 
Nodding, 458

Northern Golden, 458

Tufted, 458

Yellow Marsh, 459

Saxifrage, Saxifraga hieraciifolia, in Southern British Columbia, and its Palaeobotanical Implications, An Occurrence of the Hawkweed-Leaved, 304

Sayornis phoebe, 14 saya, 14

Scaup, Greater, 12,582

Lesser, 12,582

Scaup, Aythya affinis, Reproductive Rates Declined in Parkland Manitoba?, Have Lesser, 582

Schizachne purpurascens, 280

Schultz, R.N., 469

Scirpus spp., 103 acutus, 226

caespitosus ssp. austriacus, 280 validus, 449

Scizachyrium scoparium, 218

Scolochloa festucacea, 103

Scoter, Black, 12

Surf, 12

White-winged, 12

Scotter, G.W., Review by, 159

Screech-Owl, Western, 14

Scrub-Jay, Western, 311

Sculpin, Deepwater, 642

Fourhorn, 643

Mottled, 59

Slimy, 59

Sculpin, Myoxocephalus thompsonii, from George Lake in Whiteshell Provincial Park, Manitoba, First record of the Deepwater, 642

Scyliorhinus retifer, 475

retifer retifer, 476

Scyliorhinus retifer (Garman, 1881), New to the Canadian

Sea-blite, 456 Atlantic Ichthyofauna, The Chain Dogfish, 475

Seal, 121

$$
\text { Ringed, } 433
$$

Sears, H.J., J.B. Theberge, M.T. Theberge, I. Thornton, and G.D. Campbell. Landscape Influence on Canis Morphological and Ecological Variation in a CoyoteWolf $C$. lupus $\times$ latrans Hybrid Zone, Southeastern Ontario, 589

Sebastes caurinus, 118

emphaeus, 118

Seburn, D., Reviews by, 327,332

Seburn, D.C. Population Structure, Growth, and Age Estimation of Spotted Turtles, Clemmys guttata, Near their Northern Limit: an 18-Year Follow-up, 436

Sedge, 103,258

Alpine, 287

Awned, 285

Bent, 286

Blunt, 287

Bog, 287

Bristle, 287

Broad-wing, 453

Broad-leaved, 650

Brownish, 286

Buxbaum's, 286

Dark-brown, 286

Dunhead, 287
Few-flowered, 214

Few-seeded Bog, 287

Franklin's, 286

Garber's, 286

Glacier, 287

Goosegrass, 286

Green, 287

Hairlike, 286

Long-stalked, 650

Low Northern, 286

Northern Clustered, 285,452

Plantain-leaved, 650

Thick-headed, 287

Threadleaf, 197

Two-coloured, 286

William's, 287

Yellow, 286

Yukon, 286

Seiurus noveboracensis, 15

Selaginopsis alternitheca, 562

Selasphorus rufus, 14

Semotilus margarita, 59

Senecio indecorus, 280

jacobaea, 536

sheldonensis, 280

streptanthifolius, 280

tundricola, 280

vulgaris, 280

Sertularella polyzonias, 557

rugosa, 560

tenella, 561

Sertularia argentea, 561

cupressina, 560

fabricii, 560

latiuscula, 557

pumila, 561

similis, 560

Setophaga ruticilla, 15,206

Shad, American, 59

Shearwater, Flesh-footed, 11

Short-tailed, 11

Sooty, 11

Sheep, Dall, 7

Domestic, 309

Shepherdia canadensis, 449

Shooting Star, Few Flowered, 304

Shoveler, Northern, 12

Shrew, American Water, 7

Arctic, 83

Cinereus, 17

Dusky, 17,82

Masked, 17,87,246

Montane, 17

North American Pygmy, 82

Pygmy, 17,82

Short-tailed, 246,390

Tiny, 7

Tundra, 7

Vagrant, 82

Water, 82

Shrews (Sorex)? Revisiting the Question Using DNA Sequence Data, Are There Two Species of Pygmy, 82

Shrike, Northern, 7

Sialia currucoides, 15,110 
Sialia currucoides, and Tree Swallows, Tachycineta bicolor with Monthly Air Temperatures, Comparison of Spring Return Dates of Mountain Bluebirds, 110

Sibbaldia procumbens, 280

Sibbaldia, 295

Silene acaulis, 67 acaulis ssp. acaulis, 280 cucubalis, 260 involucrata ssp. involucrata, 280 taimyrensis, 280,449 uralensis ssp. ogilviensis, 448

Simard, R.R., 267

Siskin, Pine, 16,177

Sistrurus catenatus catenatus, 393

Sitta canadensis, 15,177 carolinensis, 177

Skua, South Polar, 13

Skunk, Striped, 30,227,348,380

Smartweed, 103

Smelt, Rainbow, 59

Smilacina trifolia, 454

Smith, B.W., C.A. Dobony, J.W. Edwards, and W.M. Ford. Observations of Long-tailed Weasel, Mustela frenata, Hunting Behavior in Central West Virginia, 313

Smith, C.M., Review by, 675

Smith, P.G.R. Winter Bird Use of Urban and Rural Habitats in Ontario, 173

Smith, T., Reviews by, 506,672

Snag, 215

Snakeroot, White, 650

Snakeweed, 645

Snipe, Common, 13,169

Snowberry, Western, 258

Soapberry, 460

Solidago canadensis var. salebrosa, 280 graminifolia, 535

missouriensis, 262

multiradiata, 280

rigida, 260

rugosa, 537

Solitaire, Townsend's, 15

Solomon's Seal, False, 650

Somateria mollissima, 12,230,608 spectabilis, 12

Somateria mollissima, Physical Condition of an Animal, Using as an Example the Common Eider, 230

Sonchus asper, 280

Sora, 7

Sorex spp., 82,246

araneus, 85

arcticus, 83

asper, 85

caecutiens, 85

cinereus, $17,84,246$

fumeus, 85

granarius, 85

haydeni, 84

hoyi, 17,82

hoyi eximius, 82

hoyi hoyi, 82

hoyi montanus, 82

hoyi thompsoni, 82

hoyi winnemana, 82

maritimensis, 83

monticolus, 17,82 pacificus, 84

palustris, 17,82

shinto, 85

thompsoni, 82

trowbridgii, 84

tundrensis, 17,85

vagrans, 82

yukonicus, 17

(Sorex)? Revisiting the Question Using DNA Sequence Data, Are There Two Species of Pygmy Shrews, 82

Sorghastrum nutans, 218

Sorrel, Green, 290

Sovada, M.A., 424

Sow-thistle, Spiny, 300

Sparganium eurycarpum, 168 minimum, 280

Sparrow, American Tree, 16,179

Brewer's, 7

Chipping, 7,169

Clay-colored, 16

Fox, 16

Gambel's White-crowned, 203

Golden-crowned, 16,206

Harris's, 203

House, 177

Lark, 16

Lincoln's, 16,169

Nelson's Sharp-tailed, 169

Rufous-collared, 206

Savannah, 16,169

Seaside, 654

Song, 7,169,179

Swamp, 169

White-crowned, 16,203,617

White-throated, 16,206

Sparrows, Z. leucophrys, in the Northwest Territories, Canada, Patterns of Nestling Feeding in Harris's Sparrows, Zonotrichia querula and White-crowned, 203

Sparrows, Zonotrichia querula and White-crowned Sparrows, Z. leucophrys, in the Northwest Territories, Canada, Patterns of Nestling Feeding in Harris's, 203

Spartina alterniflora, 654

Spears, B.L., W.B. Ballard, M.C. Wallace, R.D. Applegate, and P.S. Gipson. Coyote, Canis latrans - Rio Grand Turkey, Meleagris gallopavo intermedia, interactions, 645

Spearwort, Creeping, 292,457

Spergularia rubra, 280

Spermophilus spp., 347,365

parryii, 17

richardsonii, 30,308

tricemlineatus, 308

Sphagnum spp., 211,393,436

girgensohnii, 535

Sphagnum, 436

Sphyrapicus ruber, 14

varius, 14

Spiraea, 459

Spiraea beauverdiana, 449

Spirodesmos archimedeus, 577 interruptus, 578

Spizella arborea, 16,179

breweri, 16

pallida, 16

passerina, 16,169 
Spleenwort, Narrow-leaved, 650

Sporobolus cryptandrus, 425,645

Spruce, 203,306,362,394,566

Black, 1,72,130,362,436,532,590

Engelmann, 40,44,353

Red, 532

Sitka, 1,215

Sub-Boreal, 130

White, 1,76,130,307,353,378,532,590

Spurry, Purple Sand, 291

Squirrel, American Red, 17

Arctic Ground, 7

Flying, 193,365

Ground, 347,365

Northern Flying, 17

Red, 193,246,365

Richardson's Ground, 30,308

Thirteen-lined Ground, 308

Staniforth, J., 278

Starflower, Northern, 213

Starling, European, 15,177

Starwort, Boreal, 291

Thick-leaved, 456

Stelgidopteryx serripennis, 15

Stellaria borealis, 280,449

calycantha, 456

crassifolia, 449

graminifolia, 536

longifolia, 280

media, 280

Stephens, R.M., A.W. Alldredge, and G.E. Phillips. Aggressive Interactions of Rocky Mountain Elk, Cervus elaphus nelsoni, During the Calving Season Toward Mule Deer, Odocoileus hemionus, in Central Colorado, 316

Stercorarius longicaudus, 13

maccormicki, 13

parasiticus, 13

pomarinus, 13

Sterna aleutica, 13

caspia, 13

forsteri, 654

hirundo, 13

paradisaea, 13,309

Sterna forsteri, Eggs in Coastal North Carolina, Marsh Rice Rat, Oryzomys palustris, Predation on Forster's Tern, 654

Stewart, D.T., M. McPherson, J. Robichaud and L. Fumagalli. Are There Two Species of Pygmy Shrews (Sorex)? Revisiting the Question Using DNA Sequence Data, 82

Stichwort, Ross', 456

Stickleback, Ninespine, 59 Threespine, 59

Stickseed, Western, 297

Sticta fuliginosa, 61

Stint, Red-necked, 13

Stipa comata, 25,280 spartea, 262 spartea var. curtiseta, 260 viridula, 262

Stitchwort, Yukon, 291

Storm-Petrel, Fork-tailed, 11 Leach's, 11

Strawberry, 380
Wild, 294

Streptopus amplexifolius ssp. americanus, 280

Strix nebulosa, 14

Struik, C.L., 601

Stubblefield, W.A., 119

Sturgeon, Atlantic, 59

Lake, 541

Sturgeon, Acipenser fulvescens, in a Natural Reach of the Ottawa River, Movement of Lake, 541

Sturnella magna, 169,177 neglecta, 16

Sturnus vulgaris, 15,177

Suaeda calceoliformis, 449

Subularia aquatica ssp. americana, 280,449

Sucker, Longnose, 59 White, 59

Sullivan, R.M., 424

Sundew, Round-Leafed, 213

Surfbird, 7

Surnia ulula, 14,306

Surnia ulula, Nest on a Man-made Structure in Alaska, A Northern Hawk Owl, 306

Swallow, Bank, 15

Barn, 7

Cliff, 15

Northern Rough-winged, 15

Tree, 15,110

Violet-green, 15

Swallows, Tachycineta bicolor with Monthly Air Temperatures, Comparison of Spring Return Dates of Mountain Bluebirds, Sialia currucoides, and Tree, 110

Swan, Trumpeter, 11

Tundra, 11

Sweet-cicely, Blunt-fruited, 296

Sweet-Clover, Yellow, 168

Sweet-vetch, Boreal, 295

Swift, Vaux's, 14

Switchgrass, 218

Sycamore, 646

Sylvilagus sp., 314 floridanus, 591

Symphoricarpos occidentalis, 258

Symplectoscyphus tricuspidatus, 557

Synaptomys borealis, 6 cooperi, 247

Syncoryne mirabilis, 561

Synthliboramphus antiquus, 13

\section{Tachycineta bicolor, 15,110} thalassina, 15

Tachycineta bicolor with Monthly Air Temperatures, Comparison of Spring Return Dates of Mountain Bluebirds, Sialia currucoides, and Tree Swallows, 110

Tamarack, 590

Tamarisk, 645

Tamarix chinensis, 645

Tamias minimus, 17 striatus, 246,591

Tamiasciurus hudsonicus, 17,193,246,365

Tanacetum vulgare, 536

Tanager, Western, 7

Tanseymustard, Northern, 293

Taraxacum lyratum, 280,449

officinale, 197

phymatocarpum, 464 
Tarragon, 299

Tattler, Wandering, 13

Taxidea taxus, 308,348

Taxus brevifolia, 213 canadensis, 389

Taxus canadensis, Small Mammal Abundance and Diversity in Forests with and without Canada Yew, 389

Tea, Labrador, 213

Teal, Blue-winged, 12,227,314

Cinnamon, 12

Green-winged, 12

Tern, Aleutian, 7

Arctic, 13,309

Black, 13

Caspian, 13

Common, 13

Forster's, 654

Tern, Sterna forsteri, Eggs in Coastal North Carolina, Marsh Rice Rat, Oryzomys palustris, Predation on Forster's, 654

Texas, Den Site Activity Patterns of Adult Male and Female Swift Foxes, Vulpes velox, in Northwestern, 424

Thalictrum alpinum, 280 sparsiflorum ssp. richardsonii, 280

Thallose, 214

Theberge, J.B., 589

Theberge, M.T., 589

Thistle, 103

Thompson, F.L. Jr., 190

Thompson, I.D., Review by 155

Thornton, I., 589

Thrush, Gray-cheeked, 15

Hermit, 15

Swainson's, 15

Varied, 15

Thuiaria alternitheca, 562

argentea, 561

articulata, 562

carica, 562

kolaensis, 562

laxa, 560

thuiarioides, 561

Thuja occidentalis, 470,590 plicata, 61,212,353,566

Tiarella spp., 352

Tilia americana, 590,650

Tillaea aquatica, 458

Timothy, 168,285

Titman, R.D., 377

Tits, Great, 424

Toad, 380

American, 477

Tofieldia glutinosa, 213 pusilla, 67

Tokaryk, T.T., Reviews by, 340,341

Trefoil, Birds-foot, 295

Trichophorum caespitosum, 211

Trientalis arctica, 213

Trifolium spp., 168 pratense, 280 repens, 197

Tringa flavipes, 13 melanoleuca, 13 solitaria, 13

Troglodytes troglodytes, 15
Trout, Brook, 59

Lake, 59,642

Sea, 634

Tryngites subruficollis, 13

Tsuga spp., 362

canadensis, 590,622

heterophylla, 1,212,353,566

mertensiana, 1

Tsuji, L.J.S., 523

Tubularia crocea, 561 indivisa, 561

Tuckermannopsis chlorophylla, 62 ciliaris, 64

Turdus migratorius, 15,177,311

Turdus migratorius, Autumn and Winter Breeding Records for the American Robin, 311

Turkey, Eastern Wild, 609

Rio Grand, 645

Wild, 645

Turkey, Meleagris gallopavo intermedia, interactions, Coyote, Canis latrans - Rio Grand, 645

Turnstone, Black, 13 Ruddy, 13

Turtle, Blanding's, 415

Bog, 415

Eastern Painted, 415

Snapping, 415

Spotted, 415,436

Western Pond, 415

Wood, 377,415

Turtle, Glyptemys insculpta, at River Denys: A Second Population for Cape Breton Island, Nova Scotia, The Wood, 415

Turtle, Glyptemys insculpta, Population at the Northern Limit of its Range in Québec, Ecological Aspects of a Wood, 377

Turtles, Clemmys guttata, Near their Northern Limit: an 18Year Follow-up, Population Structure, Growth, and Age Estimation of Spotted, 436

Twinflower, 214,352,463

Twistedstalk, Clasping, 288

Tympanuchus phasianellus, 12,227,479

Typha angustifolia, 101,168

latifolia, 103

x glauca, 103

Tyrannus forficatus, 14

tyrannus, 14,169

Ulmus americana, 218,590

Uria aalge, 13

Urocyon cinereoargenteus, 468

Urosalpinx cinerea, 555

Ursus americanus, 18,113,193,236,407,566,591,621,626 americanus floridanus, 113

americanus hamiltoni, 236

americanus luteolus, 113

arctos, 18,566

horribilis, 30

maritimus, 120,433,648

Ursus americanus, Associated with Elevated Train Trestles, Mortality of Black Bears, 113

Ursus americanus, Denning Chronology and Den Site Selection in the Northeastern Cascades of Washington, Black Bear, 626 
Ursus americanus, in Difficult Locations, Methods for Capturing Free-Ranging Black Bears, 621

Ursus maritimus, in northeast Saskatchewan, Extralimital Sighting of a Polar Bear, 648

Ursus americanus, Phenotypic Variation in Skull Size and Shape Between Newfoundland and Mainland Populations of North American Black Bears, 236

Usnea lapponica, 62

Utricularia minor, 280

Uvularia grandiflora, 650

Vaccinium spp., 213,352,594

corymbosum, 395

macrocarpon, 436

myrtilloides, 535

ovalifolium, 448

oxycoccus, 436

parvifolium, 212

uliginosum, 213

vitis-idaea, 213

Vahlodea atropurpurea ssp. latifolia, 449

Van Why, K.R. and M.J. Chamberlain. Mortality of Black Bears, Ursus americanus, Associated with Elevated Train Trestles, 113

Veinotte, C., B. Freedman, W. Maass, and F. Kirstein. Comparison of the Ground Vegetation in Spruce Plantations and Natural Forest in the Greater Fundy Ecosystem, New Brunswick, 531

Veratrum viride ssp. eschscholtzii, 280

Verbena hastata, 278

Vermivora celata, 15 peregrina, 15

Vermont, Assessing an American Marten, Martes americana, Reintroduction in, 190

Vervain, Blue, 297

Vetch, American, 260

Tufted, 168

Viburnum alnifolium, 535

Vicia americana, 103,260 cracca, 168

Viola adunca, 262,449

epipsila ssp. repens, 280,449

langsdorfii, 280

nephrophylla, 280

renifolia var. brainerdii, 449

Violet, Alaska, 296

Dwarf Marsh, 296,460

Hook-spur, 460

Kidney-leaved, 460

Northern Bog, 296

Vireo gilvus, 14

solitarius, 14

Vireo, Blue-headed, 14

Warbling, 14

Virginia, Observations of Long-tailed Weasel, Mustela frenata, Hunting Behavior in Central West, 313

Virgl, J.A., S.P. Mahoney, and K. Mawhinney. Phenotypic Variation in Skull Size and Shape Between Newfoundland and Mainland Populations of North American Black Bears, Ursus americanus, 236

Vole, 245,365

Chestnut-cheeked, 17

Eastern Heather, 7

Insular, 17

Long-tailed, 7
Meadow, 17,246,466

Northern, 17

Northern Red-backed, 7

Red-Backed, 246

Singing, 7

Southern Red-backed, 390

Taiga, 7

Tundra, 7

Yellow-Nosed, 246

Vulpes fulva, 380

macrotis, $123,424,468$

velox, $30,123,424,468$

vulpes, 17,30,123,227,308,348,365,424,468,472,586,591

Vulpes velox, in Northwestern Texas, Den Site Activity Patterns of Adult Male and Female Swift Foxes, 424

Vulpes velox, White Color Phase of the Swift Fox, 468

Vulpicida pinastri, 64

Vulture, Turkey, 11

Wagtail, Black-backed, 15

White, 15

Walde, A.D., J.R. Bider, C. Daigle, D. Masse, J.-C. Bourgeois, J. Jutras, and R.D. Titman. Ecological Aspects of a Wood Turtle, Glyptemys insculpta, Population at the Northern Limit of its Range in Québec, 377

Wallace, M.C., 645

Walleye, 542

Walnut, Black, 218

Wapiti, 196

Rocky Mountain, 197

Warbler, Arctic, 7

Black-throated Blue, 389

Blackpoll, 15

MacGillivray's, 7

Orange-crowned, 15

Palm, 15

Tennessee, 7

Townsend's, 7

Wilson's, 16

Yellow, 15,206,309

Yellow-rumped, 15

Warkentin, I.G., C.E. Campbell, K.G. Powell, and T.D. Leonard. First Record of Mink Frog, Rana septentrionalis, from Insular Newfoundland, 477

Washington, Black Bear, Ursus americanus, Denning Chronology and Den Site Selection in the Northeastern Cascades of, 626

Water-hemlock, Spotted, 296

Waterthrush, Northern, 15

Waxwing, Bohemian, 15 Cedar, 15,177

Weasel, Least, 18,245 Long-tailed, 193,246,313

Weasel, Mustela frenata, Hunting Behavior in Central West Virginia, Observations of Long-tailed, 313

Webster, W.D., 654

Wheatear, Northern, 15

Wheatgrass, Crested, 168,281

Intermediate, 283

Siberian, 281

Slender, 283

Tall, 283

Western, 25,168

Whimbrel, 13

White, J.D., 311 
Whitefish, Lake, 59,642

Round, 59

Whitetop, 103

Whitlow-grass, 293

Lance-leaved, 293

Longstalk, 293

Yellowstone, 293

Whitman, J.S. Age Structure Differences in American Mink, Mustela vison, Populations under Varying Harvest Regimes, 35

Wiebe, M.O. and M.R. Lein. Response of Male Mountain Chickadees, Poecile gambeli, to Playback of Different Song Types, 76

Wiens, G., 123

Wigeon, American, 11

Eurasian, 11

Williams, A., 393

Williams, R., 634

Willis, C.K.R. and R.M. Brigham. New Records of the Eastern Red Bat, Lasiurus borealis, from Cypress Hills Provincial Park, Saskatchewan: A Response to Climate Change?, 651

Willow, 103,203

Alaska Bog, 289

Arctic, 289

Barren-ground, 289

Feltleaf, 289

Halbred, 289

Little-tree, 289

Mountain, 289

Northern, 289

Richardson's, 289

Round-leaf, 290

Snow-bed, 289,454

Variable, 289

Willowherb, Hornemann's, 296

Swamp, 296,461

Wilsonia pusilla, 16

Windels, S.K., 389

Wintergreen, Lesser, 296

Wisconsin, Death of Gray Wolves, Canis lupus, in Porcupine, Erethizon dorsatum, Dens in, 469

Wolf, 17,120,242,364,400,550,566

Alexander Archipelago, 7

Boreal Gray, 7

Gray, 7,357,419,468,469,589

Plains, 30

Red, 589

Wolf, Canis lupus, Pack Structure: Conservation Implications for Recovering Populations, Limits to Plasticity in Gray, 419

Wolf, C. lupus $\times$ latrans Hybrid Zone, Southeastern Ontario, Landscape Influence on Canis Morphological and Ecological Variation in a Coyote-, 589

Wolverine, 18,357,364,566

Wolves, Canis lupus, in Porcupine, Erethizon dorsatum, Dens in Wisconsin, Death of Gray, 469

Wood-Pewee, Western, 14

Wood-reed, Nodding, 282

Woodchuck, 7,247

Woodfern, Correll's, 651
Evergreen, 390

Woodpecker, Acorn, 311

Black-backed, 14

Downy, 14,179

Hairy, 14,177

Three-toed, 14

Woodrat, Bushy-tailed, 7

Woodsia alpina, 449

glabella, 449

ilvensis, 449

Woodsia, Northern, 451

Rusty, 451

Smooth, 451

Worm, Meningeal, 407

Wormwood, Northern, 463

Wren, Marsh, 169,654

Sedge, 348

Winter, 15

Wydeven, A.P., S.R. Boles, R.N. Schultz, and C.J.T. Doolittle. Death of Gray Wolves, Canis lupus, in Porcupine, Erethizon dorsatum, Dens in Wisconsin, 469

Xanthorion, 63

Xema sabini, 13

Yahner, R.H., 19

Yarrow, Woolly, 260

Yellow-toadflax, 298

Yellowlegs, Greater, 7

Lesser, 13

Yellowthroat, Common, 7

Yew, Canada, 389

Pacific, 215

Yew, Taxus canadensis, Small Mammal Abundance and Diversity in Forests with and without Canada, 389

Young, G.I. and R.H. Yahner. Distribution of, and Microhabitat Use by, Woodland Salamanders Along ForestFarmland Edges, 19

Yucca glauca, 645

Yucca, Plains, 645

Yukon Territory V, New Records of Vascular Plants in the, 278

Zapus hudsonius, 6 princeps, 6

Zenaida macroura, 14,177

Zhang, T.Q., 267

Zonotrichia albicollis, 16,206

atricapilla, 16,206

capensis, 206

leucophrys, 16,203,617

leucophrys gambelii, 203

querula, 203

Zonotrichia leucophrys, in the Northwest Territories, Canada, Patterns of Nestling Feeding in Harris's Sparrows, Zonotrichia querula and White-crowned Sparrows, 203

Zonotrichia querula and White-crowned Sparrows, Z. leucophrys, in the Northwest Territories, Canada, Patterns of Nestling Feeding in Harris's Sparrows, 203

Zostera marina, 655 


\section{Index to Book Reviews}

\section{Botany}

Blouin, G. An Eclectic Guide to Trees East of the Rockies, 497

Douglas, G.W., G.B. Straley, D. Meidinger and J. Pojar. Illustrated Flora of British Columbia Volumes 1-8, 329

Evans, C.L. The War on Weeds in the Prairie West: An Environmental History, 670

Kershaw, L. Ontario Wild Flowers, 330

Kirk, P.M., P.F. Cannon, J.C. David and J.A. Stalpers. Ainsworth and Bisby's Dictionary of the Fungi, 328

Lesica, P. A Flora of Glacier National Park, Montana, 159

Mitchell, R.S. and L. Danaher. Northeastern Fern Identifier, 669

More, D. and J. White. The Illustrated Encyclopedia of Trees, 496

Riley, J.L. Flora of the Hudson Bay Lowland and its Postglacial Origins, 669

Schnell, D.E. Carnivorous Plants of the United States and Canada: Second Edition, 496

\section{Environment}

Bierregaard, R.O. Jr., C. Gascon, T.E. Lovejoy, and R. Mesquita. Lessons from Amazonia: The Ecology and Conservation of a Fragmented Forest, 672

DeGraaf, R.M. Trees, Shrubs, and Vines for Attracting Birds, 160

Dixon, T.F. City Wilds: Essays and Stories about Urban Nature, 498

Donovan, T.M. and C.W. Welden. Spreadsheet Exercises in Conservation Biology and Landscape Ecology, 506

Donovan, T.M. and C.W. Welden. Spreadsheet Exercises in Ecology and Evolution, 506

D’Orso, M. Plundering Paradise, 335

Ducey, J.E. Birds of the Untamed West: The History of Birdlife in Nebraska, 1750 to 1875,336

Franklin, S.E. Remote Sensing for Sustainable Forest Management, 330

Frelich, L.E. Forest Dynamics and Disturbance Regimes: Studies from Temperate Evergreen-Deciduous Forests, 502

Higgs, E. Nature by Design, 505

Johnsgard, P.A. Great Wildlife of the Great Plains, 504

Jones, H.G., J.W. Pomeroy, D.A. Walker and R.W. Hoham. Snow Ecology: An Interdisciplinary Examination of Snow-Covered Ecosystems, 332

Larson, D., U. Matthes, and P. Kelly. Cliff Ecology: Pattern and Process in Cliff Ecosystems, 672

Morris, W.F. and D.F. Doak. Quantitative Conservation Biology: Theory and Practice of Population Viability Analysis, 507

Nadkarni, N.M. and N.T. Wheelwright. Monteverde: Ecology and Conservation of a Tropical Cloud Forest, 334

Pullin, A.S. Conservation Biology, 499

Schwartzman, D. Life, Temperature, and the Earth, 498

Song, S.J. Ecological Basis for Stand Management: A Summary and Synthesis of Ecological Responses to Wildfire and Harvesting in Boreal Forests, 500

Suzuki, D. and A. McConnell. The Sacred Balance: A Visual Celebration of Our Place in Nature, 503

Thomashow, M. Bringing the Biosphere Home, Learning to Perceive Global Environmental Change, 159
Watt, A. The Last Island: A Naturalist's Sojourn on Triangle Island, 505

Wiese, F. Seabirds and Atlantic Canada's Ship-Source Oil Pollution, 332

\section{Miscellaneous}

Arnaudin, M.P. A Bird in the Bush: The Story of the Province of Quebec Society for the Protection of Birds 1917-2002, 508

Baker, D.B. John Keast Lord: Materials for a Life, 338

Berry, M.F. The Dinosaur Filmography, 341

Browne, J. Charles Darwin, The Power of Place, 340

Debus, A.A. and D.E. Debus. Paleoimagery, The Evolution of Dinosaurs in Art, 341

Hodgson, B. Naturalists: A Journal, 342

Hodgson, B. Women Travelers: A Journal, 342

King, J. Farley: The Life of Farley Mowat, 509

Lawrence, R.D. The North Runner, 674

Nicklen, P. and H. Brody. Seasons of the Arctic, 674

Stroud, P.T. The Emperor of Nature: Charles-Lucien Bonaparte and his World, 337

Suzuki, D. When the Wild Comes Leaping Up: Personal Encounters with Nature, 339,675

\section{Zoology}

Barter, M.A., Shorebirds of the Yellow Sea: Importance, Threats and Conservation Status, 321

Behnke, R.J. Trout and Salmon of North America, 318

Brooks, R.J., D. Strickland, and R.J. Rutter. Reptiles and Amphibians of Algonquin Provincial Park, 490

Byers, J.A. Built for Speed: A Year in the Life of Pronghorn, 661

Catling, P.M., C.D. Jones and P. Pratt. Ontario Odonata. Volume 3 (including observations for the year 2001), 487

Clements, J.F. Birds of the World: A Checklist, 322

Collette, B.B. and G. Klein-MacPhee. Bigelow and Schroeder's Fishes of the Gulf of Maine, 319

del Hoyo, J., A. Elliott, and J. Sargatal. Handbook of Birds of the World. Volume 8: Broadbills to Tapaculos, 663

Downer, J. Weird Nature: An Astonishing Exploratioin of Nature's Strangest Behavior, 158

Eder, T. Mammals of Ontario, 487

Ernst, C.H. and E.M. Ernst. Snakes of the United States and Canada, 495

Fishpool, L.D.C. and M.I. Evans. Important Bird Areas in Africa and Associated Islands: Priority Sites for Conservation, 664

Gittleman, J.L., S.M. Funk, D. MacDonald, and R.K. Wayne. Carnivore Conservation, 155

Halliday, T. and K. Adler. Firefly Encyclopedia of Reptiles and Amphibians, 327

Heintzelman, D. All-Weather Hawk Watcher's Field Journal, 326

Holman, J.A. In Quest of Great Lakes Ice Age Vertebrates, 494

Houston, C.S. and W. Anaka. Birds of Yorkton - Duck Mountain, 668

Jaramillo, A. Birds of Chile, 659

Jones, K.A. Knowing Bass: The Scientific Approach to Catching More Fish, 156

Kays, R.W. and D.E. Wilson. Mammals of North America, 488 
Knott, D.L. A Spring Expedition to the Falkland Islands and Antarctica, 320

Lacey, E.A., J.L. Patton, and G.N. Cameron. Life Underground, the Biology of Subterranean Rodents, 157

Leverton R. Enjoying Moths, 327

Lieske, E. and R. Myers. Coral Reef Fishes: Indo-Pacific and Caribbean - Revised Edition, 662

Love, M.S., M. Yoklavich and L. Thorsteinson. The Rockfishes of the Northeast Pacific, 323

Manly, B.J., L.L. McDonald, D.L. Thomas, T.L. McDonald and W.P. Erickson. Resource Selection by Animals, 325

Matthiessen, P. The Birds of Heaven: Travels with Cranes, 158

Maxwell, B.A., J.K. Werner, P. Hendricks, and D.L. Faith. Herpetology in Montana: A History, Status Summary, Checklists, Dichotomous Keys, Accounts of Native, Potentially Native, and Exotic Species, and Indexed Bibliography, 491

Mecklenburg, C.W., T.A. Mecklenburg, and L.K. Thorsteinson. Fishes of Alaska, 151
Riede, K. Global Register of Migratory Species (GROMS): Database, GIS Maps, and Threat Analysis, 154

Rossi, J. and R. Rossi. Snakes of the United States and Canada: Natural History and Care in Captivity, 666

Russell, C. and M. Enns. Grizzly Heart: Living without Fear among the Brown Bears of Kamchatka, 662

Saffron, I. Caviar: The Strange History and Uncertain Future of the World's Most Coveted Delicacy, 324

Scott, J.M., S. Conant and C. Van Riper III. Evolution, Ecology, Conservation, and Management of Hawaiian Birds: A Vanishing Avifauna, 153

Shirihai, H. The Complete Guide to Antarctic Wildlife, 489

Sibley, D.A. Sibley's Birding Basics, 151

Szczerbak, N.N. Guide to the Reptiles of the Eastern Palearctic, 492

Taylor, P. The Birds of Manitoba, 660

Urquhart, E. and A. Bowley. Stonechats: A Guide to the Genus Saxicola, 667

Zug, G.R., L.J. Vitt, and J.P. Caldwell. Herpetology: An Introductory Biology of Amphibians and Reptiles, Second Edition, 493 